\title{
Do Weather Conditions Affect COVID-19 Epidemic? Evidence Based on Panel Data of Prefecture-level Administrative Regions in China
}

\section{Ruofei Lin}

Tongji University School of Economics and Management

\section{Xiaoli Wang}

Tongji University School of Economics and Management

Junpei Huang (D1830263@tongji.edu.cn )

Tongji University School of Economics and Management https://orcid.org/0000-0002-8357-0273

\section{Research}

Keywords: the COVID-19, weather conditions, causal inference, multiple linear regression, heterogeneity analysis, moderating effects

Posted Date: July 2nd, 2021

DOl: https://doi.org/10.21203/rs.3.rs-645202/v1

License: (c) (1) This work is licensed under a Creative Commons Attribution 4.0 International License. Read Full License 
1 Do Weather Conditions Affect COVID-19 Epidemic?

2 Evidence Based on Panel Data of Prefecture-level Administrative Regions in 3 China

4 Ruofei Lin ${ }^{1 \mathrm{a}}$, Xiaoli Wang ${ }^{1 \mathrm{a}}$, and Junpei Huang ${ }^{* a}$

5

6 a.School of Economics and Management, Tongji University, China

7

8 *Correspondence: Junpei Huang, School of Economics and Management, Tongji University,

9 China. Email: $1830263 @$ tongji.edu.cn; Tel:+8613524597646; postal address: Building A, Tongji

10 Building, 1500 Siping Road, Shanghai, China.

11

12 Declaration of interests: The authors declare that they have no known competing financial 13 interests or personal relationships that could have appeared to influence the work reported in this 14 paper.

15

16

17

18

1 Equal contribution. 


\section{Do Weather Conditions Affect COVID-19 Epidemic? Evidence based on}

panel data of prefecture-level administrative regions in China

21

22

23

24

25

\section{Abstract:}

Background: Similar to other infectious diseases, weather conditions may affect the COVID-19 epidemic through changes to transmission dynamics, host susceptibility, and virus survival in the environment. It's critical to explore the relationship between weather variables and the spread of the COVID-19 for understanding seasonality and the possibility of future outbreaks, developing early warning systems, infection control methods, and public health measures. However, the influence of weather change on COVID-19 edidemic is still an emerging research field, and there is still relatively limited literature available.

Objectives: Our study aims to explore the causal relationship between weather conditions and COVID-19 epidemic, the regional heterogeneity of the influence of weathner conditions in eastmiddle-west and coastal-inland, the moderating effect of diurnal temperature difference, public health measures, and public opinion on the influence of weather conditions on the epidemic to investigate the effects of these factors on the intensity of weather conditions.

Methods: First, we theoretically explain the influence mechanism of weather conditions on the epidemic based on the epidemiological triangle model. Then, we collect COVID-19-related prefecture-daily panel data in mainland China from January 1, 2020, to February 19, apply twoway fixed effect model of multiple linear regression, and also take into account other influencing factors such as population movement, public health interventions of the local government, economic and social conditions, to explore the causal relationship between weather conditions and the COVID-19 epidemic.

Results: It is found that first, there is a conditional negative linear relationship between the weather conditions and the epidemic. When the average temperature is greater than $-7^{\circ} \mathrm{C}$, there is a significant negative causal relationship between the average temperature and the growth rate of the confirmed cases. Similarly, when the relative humidity is greater than $46 \%$, the increase in the relative humidity significantly contain the epidemic. However, when the average temperature is less than $-7^{\circ} \mathrm{C}$ or the relative humidity is less than $46 \%$, the effect of weather conditions is not 
47 significant. Further, from the perspective of weather conditions, prefecture-level administrative 48 regions such as Chifeng, Zhangjiakou, and Ulanqab are more conducive to the outbreak of the 49 epidemic in winter. Then, weather conditions have a greater influence in the east than in the middle 50 and western regions, and it is better in coastal region than in the inland. Finally, increasing diurnal 51 temperature differences will improve the impact of weather conditions on the confirmed cases. In 52 dry and cold regions, higher diurnal temperature differences will increase the risk of spread of the 53 disease; Strict public health measures and good public opinion can mitigate the adverse effects of 54 cold and dry weather on the spread of the epidemic.

55 Discussion: In future research, it can adopt more detailed investigation methods. Under the legal 56 framework of privacy protection, questionnaire surveys can be carried out with patients' consent 57 to draw more accurate conclusions. At the same time, in terms of the mechanism of the role of 58 weather variables, more in-depth interdisciplinary cooperation with epidemiologists is needed to 59 study the specific impact of weather conditions on the survivability of the COVID-19 virus and 60 the immunity of susceptible populations to obtain a clearer picture and compelling conclusions.

61 Keywords: the COVID-19; weather conditions; causal inference; multiple linear regression; 62 heterogeneity analysis; moderating effects 


\section{Introduction}

In December 2019, the first case of Corona Virus Disease 2019 (COVID-19) appeared in Wuhan, Hubei Province, China, and the same cases were subsequently found in other provinces of China. On January 23, the city of Wuhan went into lockdown, and from January 23 to 30, various provinces in China initiated first-level response to major public health emergencies ${ }^{1}$. Meanwhile, within two months, the COVID-19 had spread globally, and on March 11, 2020, the World Health Organization (WHO) declared the disease a global pandemic (Allam, 2020). As of April 18, 2021, the cumulative number of confirmed COVID-19 cases worldwide has exceeded 140.332 million, and the cumulative number of deaths has exceeded 3.004 million (WHO, 2021). The rapid spread of the COVID-19 poses a colossal challenge not only to human health but also to social and economic development.

The COVID-19 is a viral respiratory illness caused by the beta-coronavirus SARS-CoV-2, and it belongs to the same coronavirus family as infectious diseases such as severe acute respiratory syndrome (SARS) and Middle East Respiratory Syndrome (MERS), and other infectious diseases which spread rapidly through aerosolized droplets and virus-contaminated hands and surfaces (Sohrabi et al., 2020). The primary mechanism of action of SARS-CoV-2 is binding to the angiotensin-converting enzyme 2 (ACE2) receptors that exist on the surface of biological membranes predominantly found in the cells of the heart, lung, arteries, intestine, and renal tissues (Lan et al., 2020). After being infected with SARS-CoV-2, the incubation period may vary from 2-14 days before the onset of symptoms, mainly affecting the lower respiratory system, which the

1 China's "National Emergency Plan for Public Health Emergencies" divides public health emergencies into four levels: particularly serious (level I), major (level II), large (level III), and general (level IV). Among them, the provincial government is responsible for emergency response and preventive control measures for public health emergencies of level I and level II. 
85 clinical manifestations are dry cough, fever, and fatigue (Keni et al., 2020). The severity of 86 symptoms varies by individuals, ranging from asymptomatic manifestations to severe life-

87 threatening symptoms, including myocardial dysfunction and acute respiratory failure (Lee et al., 88 2020). Those most at risk of severe COVID-19 manifestations are older individuals and those with 89 pre-existing conditions and multi-morbidities, particularly cardiovascular disease or diabetes 90 (Clark et al., 2020).

91 Similar to other infectious diseases, weather conditions may affect the COVID-19 epidemic 92 through changes to transmission dynamics, host susceptibility, and virus survival in the 93 environment. It's critical to explore the relationship between weather variables and the spread of 94 the COVID-19 for understanding seasonality and the possibility of future outbreaks, developing 95 early warning systems, infection control methods, and public health measures. Existing 96 epidemiological literature shows that seasonal and weather changes affect the spread of respiratory 97 pathogens. Existing literature studies the influence of weather changes on the transmission of respiratory pathogens such as influenza, SARS-COV, and MERS-COV. Weather changes have 99 the potential to facilitate the emergence of new viruses and affect epidemic transmission, morbidity, 100 and mortality (Van Doremalen et al., 2013; Barreca \& Shimshack, 2012; Sobral et al., 2020). The 101 COVID-19 is epidemiologically similar to influenza viruses in that both are highly spread through 102 the respiratory tract and cause acute infections (Cobey, 2020). However, since the COVID-19 virus 103 is very different from known viruses in terms of pathogenicity and transmission, the influence of 104 weather changes on the COVID-19 epidemic is still an emerging research field, and the existing 105 literature is still relatively limited. Moreover, there are still differences in research conclusions, 106 and it is indispensable to carry out further related research. 
The main contributions of this article are as follows. First, in this article, daily confirmed COVID19 cases reported by prefecture-level administrative regions ${ }^{2}$ of mainland China are used as the research sample, which helps to reach a more accurate conclusion. Regarding the influence of weather on the spread of COVID-19, most of the existing literature uses counrty-level samples (Iqbal et al., 2020; Huang et al., 2020) or limited region-level samples (Briz-Redón \& SerranoAroca, 2020; Thu et al., 2020). However, the country-level sample fails to capture regional differences in weather in countries with large areas and uneven population distribution such as the United States, China and Brazil.

Second, compared with the existing literature that mainly uses approaches such as Spearman's rank correlation and time series analysis (Alkhowailed et al. 2020; Bashir et al. 2020; Menebo, 2020), in this paper, we carry out multiple linear regression using panel data which is more conducive to identifying the causal relationship between variables (Angrist \& Pischke, 2008). In addition, we compare and analyze whether the relationship between weather variables and the COVID-19 epidemic is a nonlinear or conditional linear relationship, and further explore the regional heterogeneity and the moderating effects of diurnal temperature variation, public health measures, and social public opinion.

Third, different from the existing literature, we also consider the diurnal temperature difference while considering the temperature and humidity variables. So far, relevant research focuses on average temperature, minimum temperature, maximum temperature, relative humidity, and absolute humidity on the spread of COVID-19 (Huang et al., 2020; Pani et al., 2020). A few papers

\footnotetext{
${ }^{2}$ The prefecture-level administrative region is the second-level administrative region of China's administrative divisions. It is governed by the provincial administrative region, including 17 prefectures, 30 autonomous prefectures, 283 prefecture-level cities and three leagues. Prefecture-level cities (PLC) include both cities and counties, which cover rural areas with a vast land area.
} 
127 consider variables such as wind speed and precipitation simultaneously, but there is limited 128 evidence that these two variables are related to the spread of COVID-19 (McClymont \& Hu, 2021). 129 While focusing on the temperature and humidity variables, this paper for the first time considers 130 the impact of diurnal temperature differences on the spread of COVID-19. The diurnal temperature 131 difference is an essential factor affecting immunity and the incidence of infectious diseases 132 (Epstein, 2010; Cheng et al., 2014), and it is also an important variable reflecting large dimensions 133 cross north and south, significant differences in elevation between east, middle, and west, and 134 geographic differences in coastal and inland locations of China, but ignored in the existing 135 literature.

136 Fourth, compared with most of the existing literature, in addition to weather conditions, we also 137 consider control variables such as human behavior patterns, public health measures, economic and 138 social conditions, which is helpful to overcome the biased estimation caused by omitted variables. 139 The principle of the spread of COVID-19 is complicated; in addition to weather conditions, it also 140 involves some important factors. All other potential confounding factors must be controlled to 141 analyze the role of weather in the spread of COVID-19 more effectively. Regarding human 142 behavior patterns, it is related to population concentration, transportation convenience, and 143 population mobility. In this paper, we refer to the method proposed by Brockmann and Helbing 144 (2013) to calculate and incorporate the control variable "effective distance". So far, the role of 145 socioeconomic conditions in the spread of COVID-19 is unclear. This article mainly considers 146 factors such as economic development level as well as health and medical conditions. We also 147 consider the influence of the incubation period which is ignored in the previous quantitative studies 148 on the relationship between weather conditions and the spread of the virus. By incorporating some 
149 key variables ignored in the previous literature into the model in this article, the role of weather in 150 the spread of the COVID-19 can be estimated more accurately.

151 Fifth, we pay more attention to the influence of the Chinese government's stringent public health 152 measures on the estimated results than the existing literature. Different from other countries, 153 Chinese governments adopted the most comprehensive and strict measures in the world to prevent 154 and control the COVID-19 epidemic. Wuhan is not the only city that went into lockdown; many 155 local governments of other prefecture-level cities also took such a measure, however, most of the 156 existing literature does not consider the influence of these measures on analysis results. In the 157 relevant statistical analysis using China as the research sample is required to carefully consider the 158 influence of the public health measures taken by the Chinese government. This article collates and 159 evaluates public health measures taken by each prefecture-level administrative region in China, 160 including but not limited to school closures, travel restrictions, community control, social 161 distancing, quarantine, isolation, close contact tracking. In addition, we use data mining 162 technology to collect big data, such as the Baidu search index ${ }^{3}$ and Baidu migration data ${ }^{4}$

\section{2. Literature Review}

164 The relationship between climate, weather, and infectious disease epidemics has attracted people's 165 attention since 2500 years ago when Hippocrates and his followers described the relationship 166 between seasonal changes and the spread of infectious diseases (Fisman, 2007; Lloyd et al., 1983). 167 Hippocratic treatise, Airs, Waters, Places describe the influence of the environment and seasons on the constitution and instructed physicians to observe the health of a community concerning sun

\footnotetext{
${ }^{3}$ Baidu Search Index website: http://index.baidu.com/v2/index.html\#/。

${ }^{4}$ Baidu Migration website: http://qianxi.baidu.com/。
} 
169 exposure, soil, elevation, climate, and geography (Miller, 1962). During the 16th and 18th

170 centuries, interest in the effects of climate on health arose from the ability to measure

171 environmental conditions with new instruments. For example, in the United States, both Thomas

172 Jefferson and Noah Webster collected information about weather and disease (National Research

173 Council, 2001). In the mid to late 19th century and most of the 20th century, people were no longer

174 interested in the effects of seasons and climate due to the emergence of bacterial theory and the 175 development of microbiology, and turned their attention to elucidating the risk factors for 176 infectious diseases associated with host and pathogen. From the end of the 20th century to the 177 present, attention to changes in climate and weather renewed interest in understanding the impact 178 of environment, climate, and weather on the incidence of infectious diseases and other health179 related diseases (Watts et al., 2017).

Epidemiological studies find that both the mortality and virulence of the Spanish flu from 1918-

1811919 are very high, which is related to low temperature and precipitation enhancement (More et 182 al., 2020). Avian influenza A virus is the cause of Spanish flu (Taubenberger, 2006), which first 183 appeared in the autumn and winter of 1917 and spread to Europe, North America, and Asia through 184 troop mobilization and deployment during World War I (Taubenberger \& Morens, 2006). Since 185 the 1918 influenza pandemic, influenza A and B strains had continued to spread around the world. 186 There are different seasonal outbreak patterns in different weather regions and in recent history, 187 the emergence of new viruses such as the 2009 H1N1 (swine flu) pandemic, 2003 SARS, and 2012 188 MERS shows that they are related to the weather. Seasonal influenza outbreaks have prominent 189 seasonal characteristics, and the peak of the annual outbreak is consistent with winter and related 190 cold and dry weather patterns (Park et al., 2020). Seasonal outbreaks of subtropical and tropical 191 weather show different patterns, usually with persistent low-level cases in the community, with 
192 multiple outbreaks throughout the year, most commonly in the shoulder season from autumn to 193 spring (Tamerius et al., 2011; Shaman et al., 2009). The severity of these seasonal outbreaks varies, 194 and weather is related to these changes since weather changes are conducive to increasing 195 transmission or leading to increased morbidity and mortality (Liu \& Zhang et al., 2020). When a 196 cold winter is followed by a mild winter, with the weather changes, the weather changes more and 197 more, severe and early seasonal outbreaks of influenza will occur (Roussel et al., 2016; Towers et 198 al., 2013). As far as SARS is concerned, meteorological factors seem to affect the spread of the 199 virus. Tan et al. (2005) find that there is a significant correlation between SARS cases and the 200 environmental temperature 7 days before the attack, and the optimal environmental temperature 201 for SARS cases is $16 \mathrm{C}$ to $28 \mathrm{C}$. Lin et al. (2006) find that the incidence of SARS at lower 202 temperatures is 18 times higher than that at higher temperatures and respiratory diseases are 203 common in colder environments. At higher weather temperatures, the virulence of the pathogen 204 will worsen because they may not withstand environmental changes. Gardner et al. (2019), based 205 on the cases of MERS in Saudi Arabia, find that MERS is more likely to occur in relatively cold 206 and dry conditions, similar to the seasonal patterns of other respiratory diseases in temperate 207 regions. Altamimi \& Ahmed (2020), based on the case of MERS in Riyadh, find that the incidence 208 of MERS is affected by weather conditions, and it shouws an upward trend from April to August. 209 High temperature and low relative humidity are the reasons for the increase in MERS cases. 210 Although there are some achievements regarding the relationship between weather conditions and 211 emerging infectious diseases, the relevant literature is still quite limited (Paraskevis et al., 2020).

212 Research on the influence of weather conditions on the spread of COVID-19 is in its infancy, and 213 there are few related papers. Most of the literature focuses on temperature and humidity while a 214 few involve other weather conditions such as wind speed and precipitation. However, the evidence 
215 for the correlation between wind speed and precipitation and the spread of COVID-19 is limited 216 (McClymont \& Hu, 2021). Therefore, we mainly focus on two weather conditions, that is 217 temperature and humidity.

218 Regarding the relationship between temperature and the COVID-19 epidemic, the existing 219 literature explores the role of minimum temperature, maximum temperature, or average 220 temperature as variables for weather conditions, and the research conclusions are entirely different.

221 Many works of literature conclude that temperature negatively correlated with the spread of 222 COVID-19, that is the higher the temperature, the fewer people infected. Meyer et al. (2020) study 223 samples from 100 countries worldwide and find that when the temperature rises above $-15^{\circ} \mathrm{C}$, 224 there is a significant negative correlation between daily temperature and daily global cases. Shi et 225 al. (2020) take samples of COVID-19 cases from 30 provincial administrative regions in China 226 and find that the incidence rate varies with temperature, where the higher the temperature, the 227 lower the incidence of the COVID-19; conversely, the lower the temperature regions, the more 228 people will be infected. Liu \& Zhou et al. (2020) use 30 provincial capital cities in China as the 229 research sample, and they find the average temperature significantly negatively correlated with the 230 number of the COVID-19 cases. Nevels et al. (2021) believe the temperature was negatively 231 correlated with the transmission rate of COVID-19 in the early stage of the outbreak in Wuhan. 232 However, some literature concludes that temperature positively correlated with the spread of 233 COVID-19. For example, Iqbal et al. (2020) take 210 countries and territories worldwide as a 234 sample and conclude that the average temperature and daylight hours have shown a positive 235 association towards the spread rate of COVID-19. Islam et al. (2021) take cases from 206 236 countries/regions as samples and find that the COVID-19 cases positively correlated with the 14237 day lag temperature. Paniet al. (2020) conduct a study on cases in Singapore and conclude that the 
average temperature and minimum temperature significantly positively correlated with the number of both new cases and the total cases. There is also some literature suggesting that temperature does not correlate with the spread of COVID-19 or the correlation relationship is uncertain. In the research of Jahangiri et al. (2020) on Iran and Briz-Redón \& Serrano-Aroca (2020) on Spain, they find no correlation between temperature and the spread of COVID-19. Hossain et al. (2021) study cases in South Asian countries such as Afghanistan, Bangladesh, India, Pakistan, and Sri Lanka and conclude that the influence of temperature on the COVID-19 epidemic is different in different countries with some positive and other negative correlations.

Regarding the relationship between humidity and the spread of COVID-19, the existing literature studies the influence of absolute humidity or relative humidity as the variable of weather conditions, and the research conclusions are also entirely different. Most literature reports a negative correlation between humidity and the spread of COVID-19. For example, Wu et al. (2020) collect data from 166 countries other than China and find a negative correlation between relative humidity and the number of new cases and deaths per day, where a $1 \%$ increase in relative humidity leads to a $0.85 \%$ decrease of the new cases per day and a $0.67 \%$ decrease of new deaths. Qi et al. (2020) study cases in 30 provincial administrative regions in China and conclude that relative humidity significantly negatively correlated with the number of cases. When the temperature is between $5.04^{\circ} \mathrm{C}$ and $8.2^{\circ} \mathrm{C}$, for every $1 \%$ increase in relative humidity, daily cases will decrease by $11-22 \%$. Zhu et al. (2020) collect daily new cases in 8 hard-hit areas in 4 countries in South America and find that absolute humidity significantly negatively correlated with daily confirmed cases. While some other literature believes that humidity positively correlated with the spread of the COVID-19. For example, Chien and Chen (2020) study 50 counties in the United States with the highest cumulative confirmed cases and find that relative humidity has a significant positive 
261 correlation with the cumulative cases. Alkhowailed et al. (2020) report a weak positive correlation

262 between average relative humidity and new cases in Saudi Arabia. Other literature suggests that

263 humidity is not related to the spread of COVID-19 or cannot be determined to be related. Meyer

264 et al. (2020) collect national data on the COVID-19 cases as of March 17, 2020, and find no

265 correlation between relative humidity and the number of the COVID-19 cases. Pan et al. (2021)

266 collect cases from 202 locations in 8 countries and conclude that weather conditions such as

267 temperature, relative humidity, wind speed, and ultraviolet rays significantly did not correlate with

268 the COVID-19 cases. Pahuja et al. (2021) study the number of cases in New Delhi, India, and did

269 not observe a correlation between the number of cases and humidity or wind speed neither.

270 Regarding the relationship between weather conditions such as temperature and humidity and the

271 COVID-19 epidemic, the different conclusions reached by existing literature mainly due to two

272 aspects. The first reason is the problem of sample selection. Most of the existing literature selects

273 national-level samples or limited large region and city-level samples. As Polgreen \& Polgreen

274 (2018) point out, to accurately verify the relationship between weather conditions and infectious

275 diseases, it is needed not only the geographic area where the cases occurred but also a control

276 group without infectious diseases. Selecting geographic areas with cases as samples for statistical

277 analysis is prone to sample selection bias (Chen and Astebro, 2001). Moreover, national sample

278 fails to capture regional differences in weather conditions between countries with large areas and

279 uneven population distribution, such as the United States, China, and Brazil.

280 The second reason is the problem of statistical analysis methods. In our search of 28 studies using 281 statistical models up to March 2021, most of them used Spearman's rank correlation, time series 282 analysis. Pearson's correlation, generalized linear model (GLM), Generalized Linear Mixed Model 283 (GLMM), and Generalized Additive Model (GAM) are used once. So far, these methods are more 
284

285

286

287

288

289

290

291

292

293

294

295

296

297

298

299

300

301

302

303

304

305

306

common in research projects on the relationship between weather and disease outside the field of infectious diseases, while there are few pieces of research in infectious diseases, and the methods are not yet mature (Polgreen \& Polgreen, 2018). First, the limitations of these methods are apparent. For example, the time series regression model is built on the premise that the data satisfy linearity and staticity, and relies on a large amount of uninterrupted time series data. However, the COVID19 case data is not necessarily linear, and the data is difficult to satisfy the requirements of uninterrupted time series. The applicable conditions of Spearman's correlation are that there are no repeated values in the data, and the two variables have a monotonic relationship, and the Spearman model calculates the grade correlation coefficient, based on the rank, which discards some vital information of the original data (Owen \& Anil, 2009). The application of Pearson correlation has strict requirements on data, that is, normal distribution, but the actual situation of the COVID-19 cases is challenging to meet this requirement. Second, there is Omitted Variable Bias in most of the literature. The spread of COVID-19 is closely related to population movement and public health interventions. Studies show that public health interventions have a much more significant impact than the weather and climate variables (Oliveiros et al., 2020). Paraskevis et al. (2020) conclude that the seasonality of COVID-19 is very different from the common cold coronavirus or influenza, and in the absence of public health measures, climatic conditions cannot alleviate the spread of COVID-19. Most of the existing research does not consider the influence of population mobility or public health intervention. However, the intervention intensity of public health measures varies significantly from country to country, and most of the literature lacks a relatively accurate assessment of this difference. The omission of this critical variable can lead to a significant bias in the estimation results (Angrist \& Pischke, 2008).

\section{Conceptual Framework}


307 The existing literature on the relationship between weather conditions and the spread of COVID30819 is preliminary, and the conclusions are opposite. However, based on the epidemiologic triangle 3095 and related literature on respiratory viruses similar to the COVID-19, we believe that weather 310 conditions such as temperature and humidity may impact the spread of COVID-19. First, weather 311 conditions determine the viability and persistence of the virus in the air and on the surface 312 (Aboubakr et al., 2021). Generally speaking, the continuous increase of COVID-19 and similar 313 viruses is related to low temperature and low relative humidity. Chan et al., 2011 report that SARS 314 can survive for more than 5 days on smooth surfaces at temperatures of $22-25^{\circ} \mathrm{C}$ and relative 315 humidity of 40-50\%. Some literature also proves that temperature and humidity are known factors 316 affecting the survival of SARS, MERS, and influenza (Otter et al., 2016). A recent study shows 317 that the COVID-19 can survive on glass, stainless steel, and paper currency for 28 days at the 318 optimal temperature of $20^{\circ} \mathrm{C}$ while reduce survival time to 24 hours at $40^{\circ} \mathrm{C}$ (Riddell et al., 2020). 319 Outside of the optimal ranges, the viability of the virus is limited, but it is sufficient to spread since 320 people lack an adaptive immune response to the previously unknown coronavirus .

321 Second, there is an influence of weather conditions on the susceptibility of the host. Cold and dry 322 air suppresses the innate immune response by damaging the mucosa and slowing mucociliary 323 clearance (Lowen et al., 2007). The innate immune response is essential to prevent initial infection, 324 inhibit viral replication, and regulate the severity of immune response and inflammation (García, 325 2020). Exposure to the cold environment may cause hormonal changes, which directly or indirectly 326 alters the immune system (van der Lans et al., 2015). Some researchers find that low temperature

\footnotetext{
${ }^{5}$ All infections involve pathogens, hosts, and the environment.
} 
327 is associated with decreased lung function and worsened condition in patients with chronic 328 obstructive pulmonary disease (Donaldson et al., 1999).

329 Third, weather conditions will affect human behavior patterns. Dai \& Zhao (2020) point out that 330 in subtropical or tropical climates, due to increased humidity and heat, people usually gather in 331 air-conditioned buildings with reduced indoor ventilation and airflow, which may increase the risk 332 of virus transmission. Menebo (2020) holds that increased sunshine and warmer weather lead to 333 an increase in the number of people gathering in outdoor spaces which enhances the risk of the 334 COVID-19 transmission. The conceptual framework of this article is as follows:

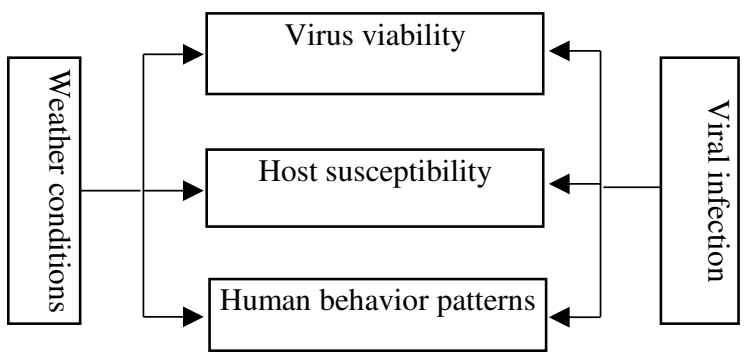

Figure 1 mechanism of the influence of weather conditions on the COVID-19 epidemic

337 China is the first country to be attacked, and cases occurred in all provincial-level administrative regions and most prefecture-level administrative regions (as of the end of February, there were 19 339 prefecture-level administrative regions without outbreaks). China's territory spans the tropical, 340 temperate, and frigid zones from south to north, where the Qinling-Huaihe line as a geographic 341 boundary is the $0{ }^{\circ} \mathrm{C}$ isotherm in January. The average temperature in January is above 0 in the 342 south of the Qinling and below 0 in the north. There is a big difference in temperature between the northern and southern prefecture-level administrative regions. In January 2020, the average high 344 temperature in the northern city of Heihe is $-13^{\circ} \mathrm{C}$, and the average low temperature is $-25^{\circ} \mathrm{C}$, while 345 in the southern city of Sanya is $25^{\circ} \mathrm{C}$ and $16^{\circ} \mathrm{C}$, respectively, with a difference of $38^{\circ} \mathrm{C}$ and $41^{\circ} \mathrm{C}$. 346 In February 2020, the average high temperature in Heihe is $-9^{\circ} \mathrm{C}$, and the average low temperature 
347 is $-21^{\circ} \mathrm{C}$, while in Sanya is $27^{\circ} \mathrm{C}$ and $19^{\circ} \mathrm{C}$, respectively, with a difference of $36^{\circ} \mathrm{C}$ and $40^{\circ} \mathrm{C}$. In

348 China, January is usually the coldest month, but the temperature starts to rise in February and rises

349 sharply in March when spring begins when most of the country's temperature is above 0 , and the

350 temperature difference between regions is greatly reduced. In March 2020, the highest temperature

351 in Sanya reaches $30^{\circ} \mathrm{C}$, and that in Heihe is $14^{\circ} \mathrm{C} .{ }^{6}$ The regional temperature difference between

352 the north and south of China provides an excellent case for studying the influence of weather

353 condition on the COVID-19 epidemic, especially the large temperature difference between the

354 cold temperature below 0 in the north and the warm temperature above 0 in the south in January

355 and February. At the same time, China also adopted stringent public health measures and basically

356 brought the epidemic under control within more than two months. On February 12, the number of

357 new cases reached a peak of 15,152 and on February 19, the number nationwide dropped to 394

358 when the number of cases in most provinces is already low except for a few provinces such as

359 Hubei. There were no new confirmed cases on March 20 for the first time, after which, most

360 provinces had no new cases or only sporadic cases. Considering the temperature and the number

361 of confirmed cases simultaneously, in this paper, we select the data set of all prefecture-level

362 administrative regions in mainland China from January 1 to February 19, 2020, as the research

363 sample.

\footnotetext{
${ }^{6}$ The above temperature data is inquired from the National Meteorological Information Center (NIMIC) website, http://data.cma.cn.
} 


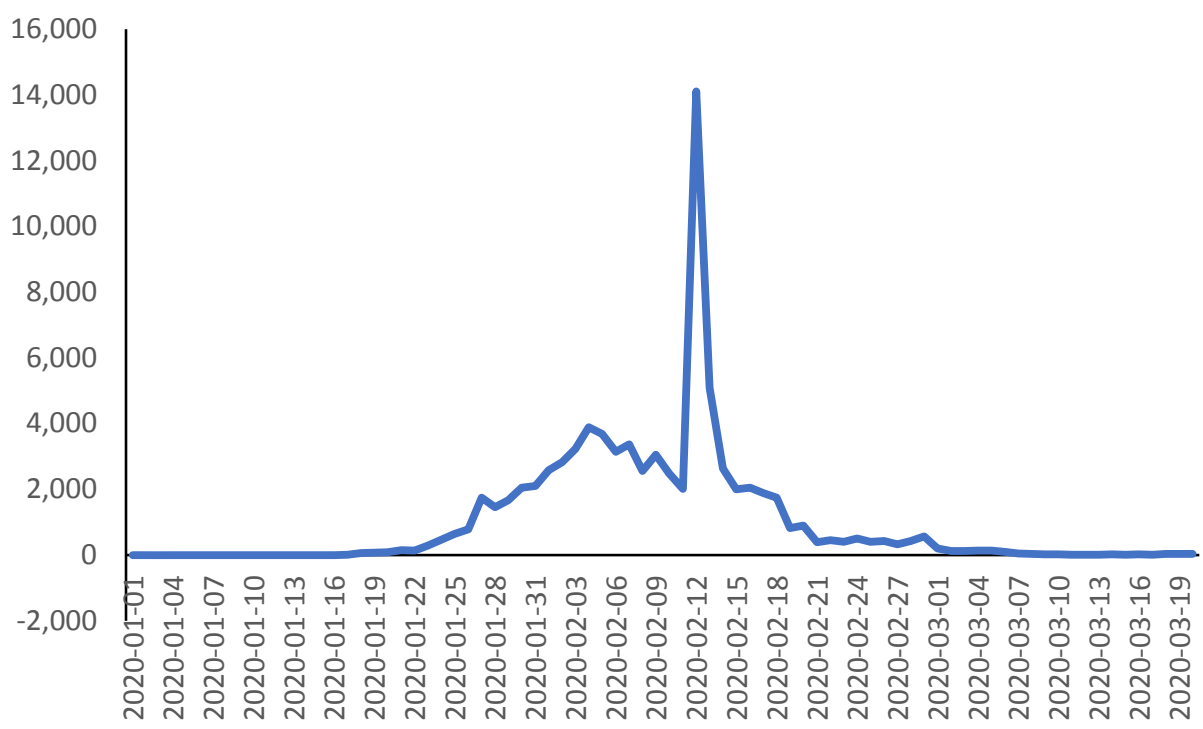

Figure 2 New confirmed cases of the COVID-19 in China from January 1, 2019 to March 20, 2020

366 Based on the above analysis,we proposes the research hypotheses as follows.

367 Hypothesis 1: There is a negative causal relationship between the average temperature and the 368 COVID-19 epidemic;

369 Hypothesis 2: There is a negative causal relationship between the relative humidity and the 370 COVID-19 epidemic.

\section{Research Design}

\section{$372 \quad 4.1$ Empirical Model and Variable}

373 Based on the theoretical analysis above, we apply econometrics approach and empirically test the influence 374 of weather condition on the epidemic using panal data of mainland China. Econometric approach is 375 commonly used to measure the effects of a factor on economic growth. Similar to early COVID-19 376 infections, economic output generally increases exponentially with a variable rate that can be affected by 377 policies and other conditions (Hsiang et al., 2020). Therefore, it is appropriate to apply econometrics techniques to analyze the influence of weather condition on the outbreak of the epidemic. As mentioned 
379 above, compared to statistical methods such as Pearson's correlation coefficient to identify the 380 correlation between weather and COVID-19 (Alkhowailed et al. 2020; Bashir et al. 2020; Menebo, 381 2020), the multiple linear regression approach of panel data is conducive to overcoming the 382 limitations of the time-series regression and Spearman regression model adopted by most existing 383 literature, and pays more attention to identifying the causal relationship between the variables 384 (Angrist, 2008), that is, whether changes in temperature and humidity lead to changes in the 385 epdidemic. The key of causal inference is to control the observable factors that interfere with the 386 causal relationship. In order to avoid biased estimators led by omitting variables, we adopt the 387 two-way fixed effect model to control the time-invariant individual heterogeneity and the 388 individual-invariant time heterogeneity. The empirical model is as follows:

$$
\text { rate }_{i t}=\alpha+\beta_{1} A T_{i t}+\beta_{2} \mathrm{RH}_{i t}+\beta_{3} \text { Measure }_{i t}+\beta_{4} \text { bed }_{i t}+\beta_{5} \text { pop }_{i t}+\beta_{6} \text { distance }_{i t}+t+\delta_{i}+\delta_{t}+\varepsilon_{i t}
$$

391 Where, rate $_{i t}$ is the explained variable, representing the actual cumulative case growth rate of 392 city $i$ on date $t$.

$$
\text { Actual cumulative case growth rate }_{i t}=\left(\text { Actual case }_{i t}-\text { Actual case }_{i t-1}\right) / \text { Actual case }_{i t-1}(2)
$$

394 If there are no cases on the current day, actual case $_{i t-1}=0$, let

395 Actual cumulative case growth rate it $=0$.

396 If there are no confirmed cases on that day, then Actual cumulative cases $_{i t-1}=0$, Let the 397 cumulative case growth rate be 0.

398 Considering that the average incubation period of COVID-19 is 5.2 days (Li et al., 2020), we 399 take the fifth lead of reported cases as the proxy variable of the actual cases, namely:

$$
\text { reported } \text { case }_{i t}=\text { actual }_{\text {case }} i t+5
$$


401 Taking Chengdu, China as an example, the number of reported cumulative confirmed cases in 402 this city on February 1, 2020, is 73. Considering the incubation period of 5 days, 73 should be 403 the number of real cumulative cases five days previously, i.e., on January 27, 2020, while the 404 number of real cases on February 1 should be the cumulative number of reported cases on 405 February 6 (see table 1$)$.

Table 1 Conversion between the actual cases and the reported cases

\begin{tabular}{|c|c|c|}
\hline Date & Reported & Actual \\
\hline 24-Jan & 12 & 59 \\
\hline 25-Jan & 22 & 69 \\
\hline 26- Jan & 33 & 72 \\
\hline 27- Jan & 37 & 73 \\
\hline 28-Jan & 46 & 77 \\
\hline 29- Jan & 59 & 87 \\
\hline 30- Jan & 69 & 92 \\
\hline 31- Jan & 72 & 97 \\
\hline $1-\mathrm{Feb}$ & 73 & 102 \\
\hline $2-\mathrm{Feb}$ & 77 & 109 \\
\hline $3-\mathrm{Feb}$ & 87 & 120 \\
\hline 4-Feb & 92 & 123 \\
\hline $5-\mathrm{Feb}$ & 97 & 124 \\
\hline
\end{tabular}




\begin{tabular}{|l|l|c|}
\hline 6-Feb & 102 & 125 \\
\hline
\end{tabular}

408 Since the existing literature shows limited evidence for the correlation between wind speed, 409 precipitation, and other weather variables (McClymont \& Hu, 2021), temperature and humidity 410 are selected as weather condition variables. $A T_{i t}$ denotes the average temperature of city $\mathrm{i}$ on date $411 \mathrm{t}$, and $R H_{i t}$ denotes the relative humidity of city $\mathrm{i}$ on date $\mathrm{t}$.

412 Measure $_{i t}$ denoes the total score of public health measures of the city $\mathrm{i}$ on date $\mathrm{t}$, which uses 413 detailed assessment data from all prefecture-level regions. Compared with other countries, China's 414 public health measures are stringent, but the central government does not uniformly stipulate them. 415 Instead, regional governments decide when to start and what measures to take based on local 416 conditions, therefore, there are significant differences between regions. In this paper, we conduct 417 a very detailed evaluation through a manual collection of data. Hanage (2020) concludes that the 418 comprehensive intervention measures implemented in China successfully alleviate the spread of 419 COVID-19, especially in the early stages of the outbreak, so we include the factor into the model 420 total score of public health measures.

421 The population movement is measured by effective distance which is proposed by Brockmann \& 422 Helbing (2013) as a new concept. They believe that population movement does not depend on the 423 geographical distance between regions but the convenience of mobility. In this paper,we calculate 424 the effective distance and incorporate it in the model (distance $\left.{ }_{i t}\right)$.

425 Regarding the socio-economic development, we take medical conditions, economic development 426 level, and population size into consideration and also take number of beds in medical institutions 427 (bed), GDP per capita (pergdp), and population size (pop) as control variables. Although the data 
structure is a wide panel, the time span is relatively long, and the development of the epidemic itself has a time trend, so we introduced the time trend " $t$ " to control the variation trend of the explained variable over time. It is common in econometric studies to consider time trend in modeling. For example, when exploring the influence of economic development on the degree of democracy, Markus Bruckner et al. (2011) also introduced t into the econometric model considering that democratic development itself has time trend. $\delta_{i}$ is a region fixed effect to control the characteristics of provinces constant over time, $\delta_{t}$ is a time fixed effect to control to control the time factors that do not vary from individual to individual. $\varepsilon_{i t}$ is error term, we use clusterrobust standard error to estimate the standard deviation (Cameron \& Miller, 2015).

\subsection{Data}

In the research data in this article, the maximum and minimum temperature data in selected areas comes from website of the National Meteorological Information Center (NMIC) ${ }^{7}$. the cumulative confirmed case of came from the official release of the National and local Health Commission. The original data on public health interventions is collected according to the information or announcements issued by the prevention and control headquarters of the prefecture-level administrative districts. The data of population movement used to calculate the effective distance comes from Baidu Migration, which is the data of migration and outflow between different regions collected by Baidu inc using big data technology. population size, GDP per capita, and number of beds in medical institutions are from the China City Statistical Yearbook. Due to the lack of statistical data in some prefecture-level administrative regions in the China City Statistical Yearbook, the number of regions returned to use was finally 279 after being eliminated.

\footnotetext{
${ }^{7} \mathrm{NMIC}$ website query, http://data.cma.cn.
} 


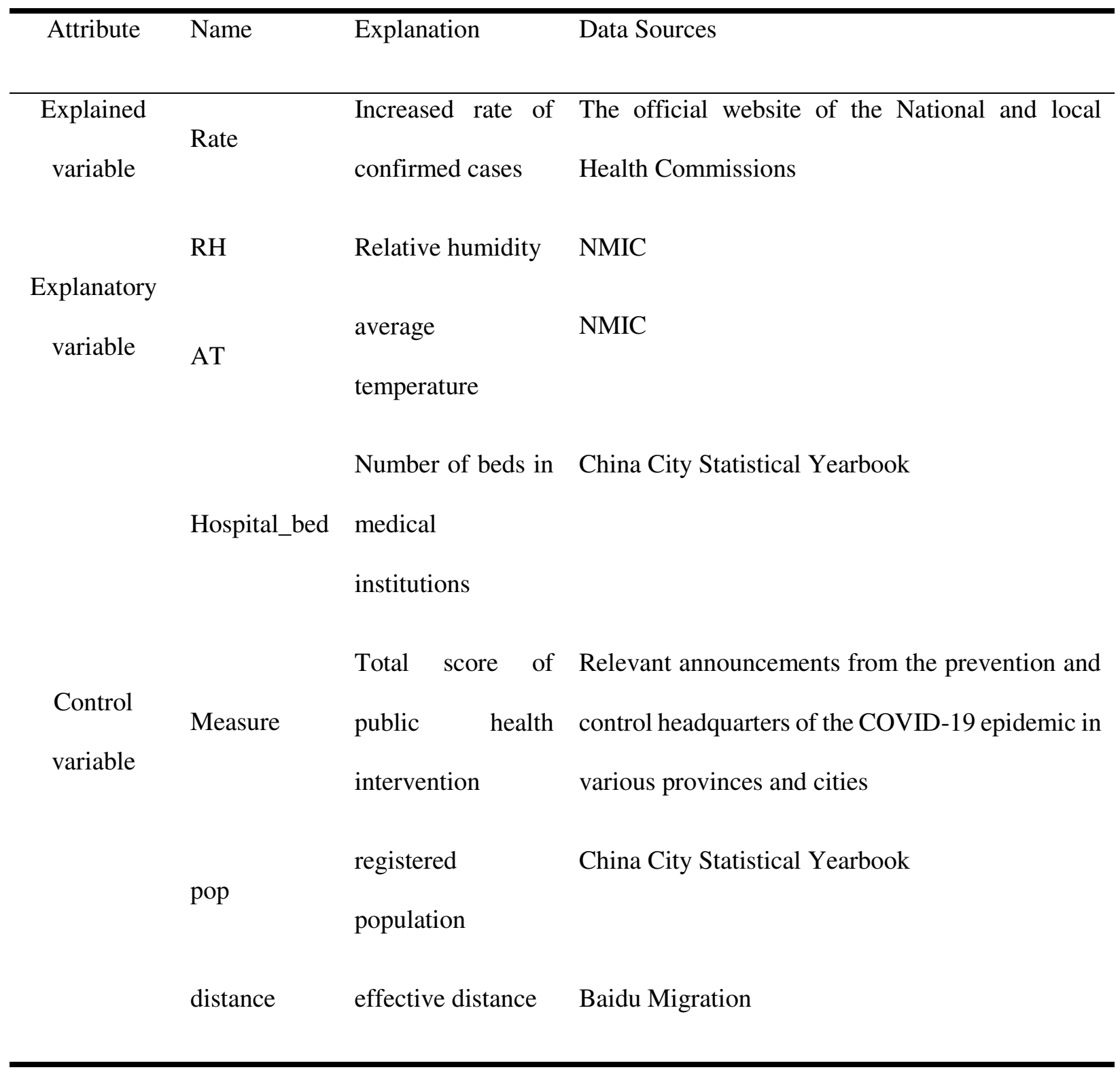

\section{$451 \quad 4.3$ Calculation of Major Variables}

\section{2 a. Weather Variables of prefectural-level administrative regions}

453 We take average temperature (AT) which is the average value of the highest temperature (HT) and 454 the lowest temperature (LT), as the proxy variable of temperature: 
456 Figure 3 shows the distribution of the mean of the average temperature from January 1 to February 45719,2020 , from which it can be seen that the average temperature decreases in a step-like manner 458 from south to north.

459 The calculation method relative humidity $(\mathrm{RH})$ is as follows.

460

461

$$
\mathrm{RH}=E_{S} / E_{0}
$$

$$
E_{0}=6.11 * 10^{\frac{7.5 T_{c}}{T_{c}+237.7}}
$$

$$
E_{S}=6.11 * 10^{\frac{7.5 T_{d}}{T^{+237.7}}}
$$

463 Where $T_{c}$ is the air temperature (degrees Celsius), $T_{d}$ is the dew point temperature (degrees 464 Celsius). Figure 4 shows the distribution of the mean of the relative humidity from January 1 to 465 February 19, 2020. It can be seen that the relative humidity in the southeast coastal area is higher, 466 and it shows a decreasing trend in the northwest direction. 


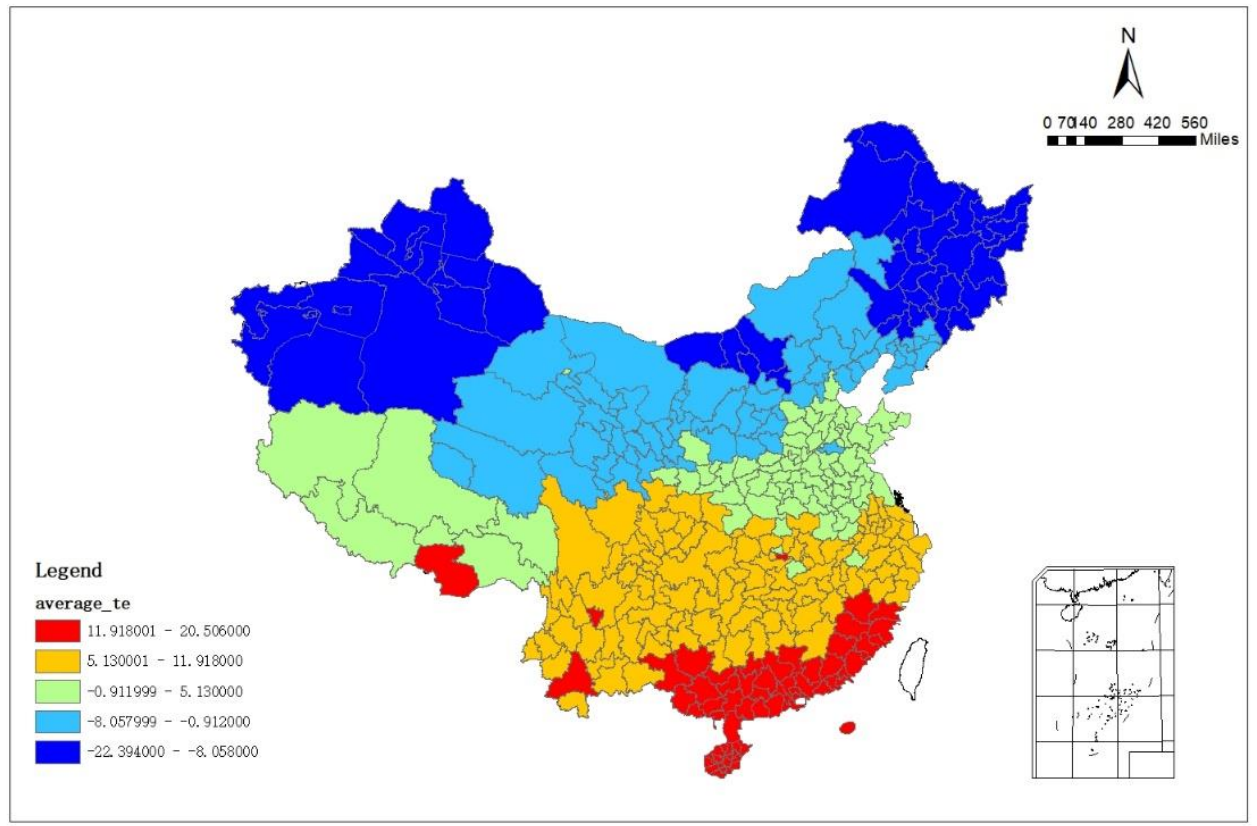

467

468

Figure 3 Mean of the Average temperature in China from January 1 to February 19, 2020

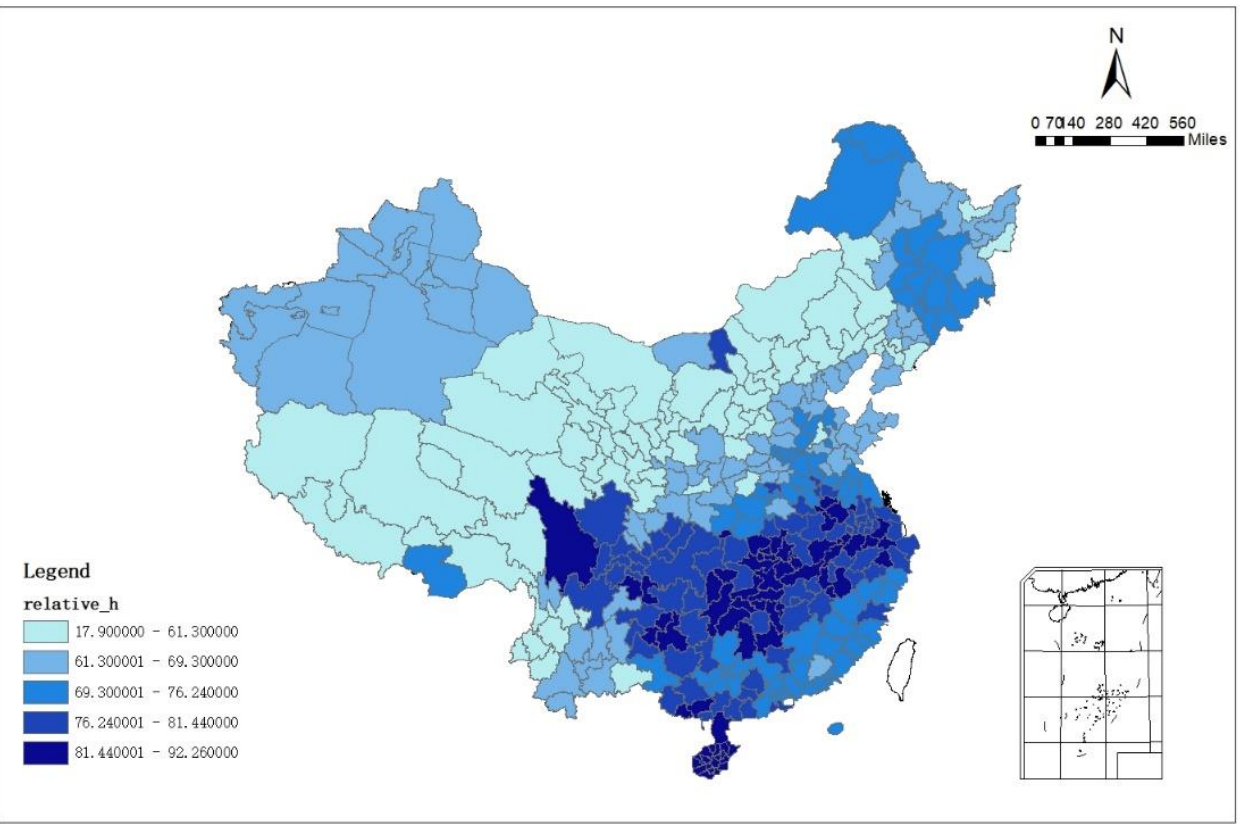


471 b. Total scores of public health intervention measures in prefecture-level administrative 472 regions

473 We construct scoring data as proxy variable for public health intervention measures. According to

474 the public health intervention measures taken by various prefecture-level administrative regions, 475 we summarize 15 specific items (see table 3 ), each with a score of 1 . Scoring starts until the 476 measure is canceled. For example, on January 21, Shanghai began to implement "quarantining the 477 contacts for 14 days, then the score of Shanghai from January 21 is 1 . On January 24, Shanghai 478 began to implement "closing part of the indoor urban public places", then the socre of Shanghai is 479 added another 1 point since January 24 , and so on, and finally, the points will be added up. The 480 total score of public health intervention measures on February 19, 2020 is shown in the figure 5.

Table 3 Items of Public Health Intervention Measures

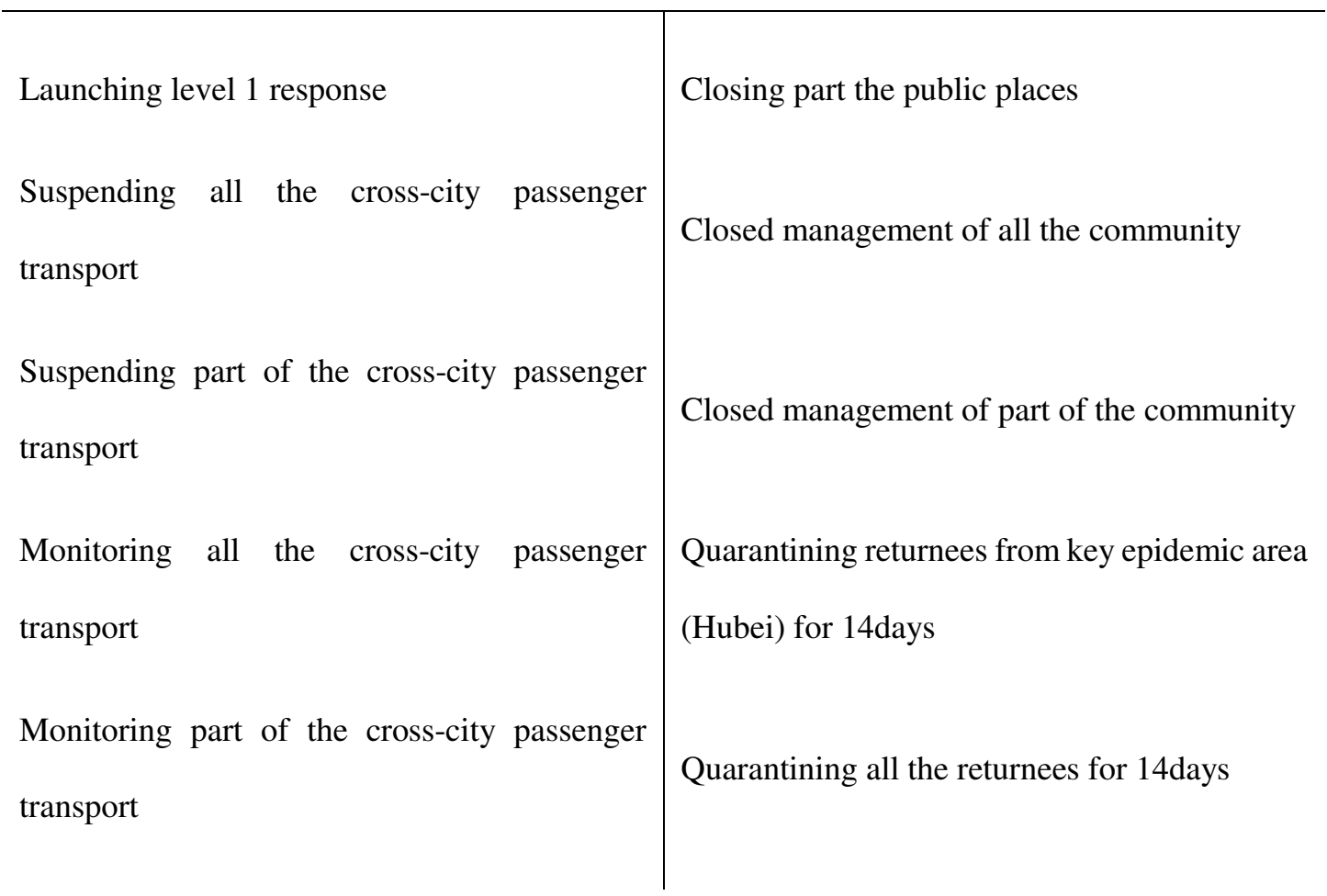




\begin{tabular}{l|l} 
Suspending all the public transport & Quarantining the contact for 14days \\
Suspending part of the public transport & Isolating and testing the suspected \\
Closing all the public places & \\
\hline
\end{tabular}

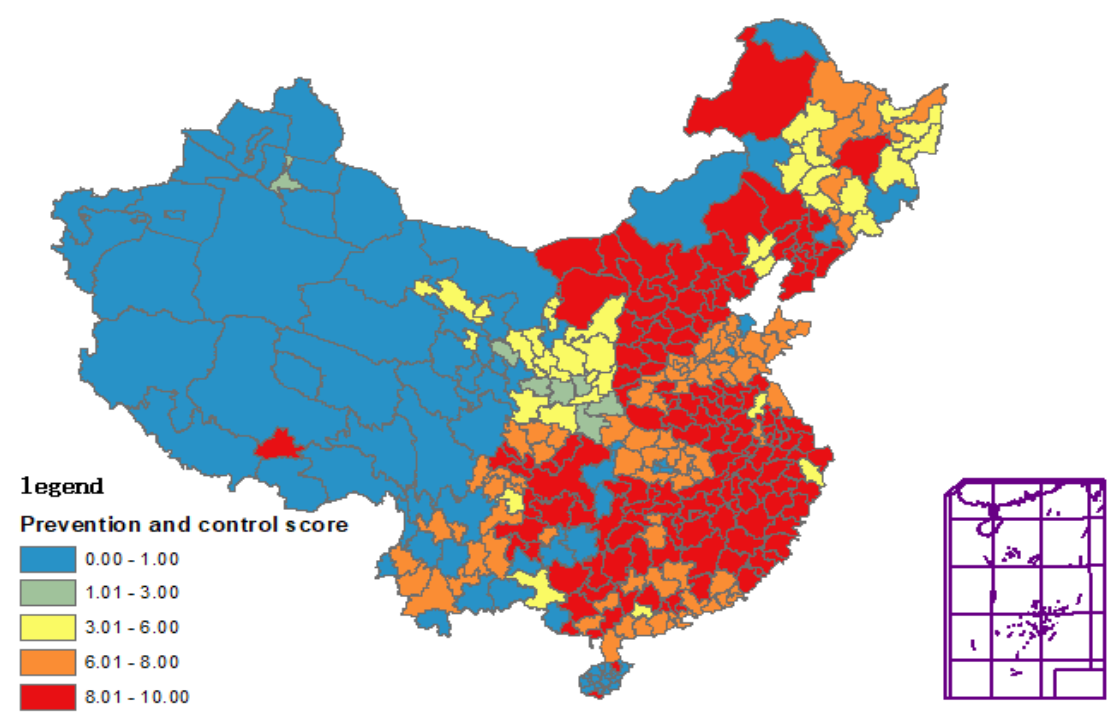

Figure 5 Total Scores of Public Health Measures in China on February 19, 2020

\section{c. Effective distance to Wuhan}

486 We draw on the concept of effective distance proposed by Brockmann and Helbing (2013) to better

487 reflect the population movement between regions. They believe that the spread of the disease has

488 nothing to do with the geographic distance between cities but is closely related to the effective

489 distance. The effective distance between cities is the length that passengers choose between the

490 various alternative routes from city i to city $\mathrm{j}$. Passengers can be regarded as random particles, and

491 they can visit the surrounding cities randomly according to traffic flow. After arriving at the next

492 city, according to the traffic flow, it is converted into the probability of visiting the neighboring 
493 cities of the next city. Finally, the path that the particle are most likey to choose from city i to city

$494 \mathrm{j}$ is the most probable path, and the length of the most probable path is the effective distance. This

495 effective distance of probability is related to population movement and traffic convenience. The

496 shorter the effective distance, the greater the probability of spreading the epidemic to the region,

497 the greater the probability of increasing the input cases, and the earlier the outbreak time of the

498 large-scale epidemic. The calculation of effective distance draws on the improved method of Lin

499 et al. (2020). Assuming that $P_{m n}$ is the proportion of the population from node $\mathrm{N}$ to node $\mathrm{M}$, since

500 the effective distance is cumulative, and the probability of multi-segment paths is calculated by

501 multiplication, so $P_{m n}$ take the logarithm, that is, the effective distance from node $\mathrm{N}$ to node $\mathrm{M}$ is

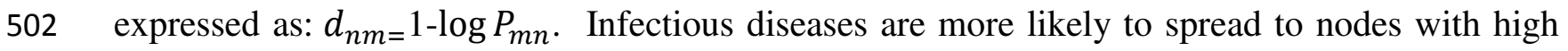

503 connectivity in the network, where this kind of inequality is represented by $d_{m n} \neq d_{n m}$. The

504 effective propagation distance depends only on the topological characteristics of the network, that

505 is, the matrix P. In this paper, the probability in the effective distance $P_{m n}$ (the proportion of the

506 traffic flow from node $\mathrm{M}$ to node $\mathrm{N}$ to the total traffic flow from all nodes to node $\mathrm{N}$ ) calculated

507 using the traffic flow from the Baidu migration data. When there are multiple paths between two

508 nodes, we can traverse all the paths $\Gamma=\left\{n_{1}, \Lambda, n_{L}\right\}$, and take the shortest one of the effective

509 distance as the final path length between two nodes, ${ }_{m n}=\min _{\Gamma} \lambda(\Gamma) D_{m n} \neq D_{n m}$. Among random paths

510 starting at node $\mathrm{N}$ and ending at node $\mathrm{M}$, the path closest to a straight line has the most significant

511 probability and the shortest effective distance. Starting from a selected starting node N, the shortest

512 paths to other nodes can form a shortest-path tree (Brockmann \& Helbing, 2013). The effective

513 distance between Wuhan and each other prefectural-level administrative regions on February 19, 5142020 is shown in Figure 6. 


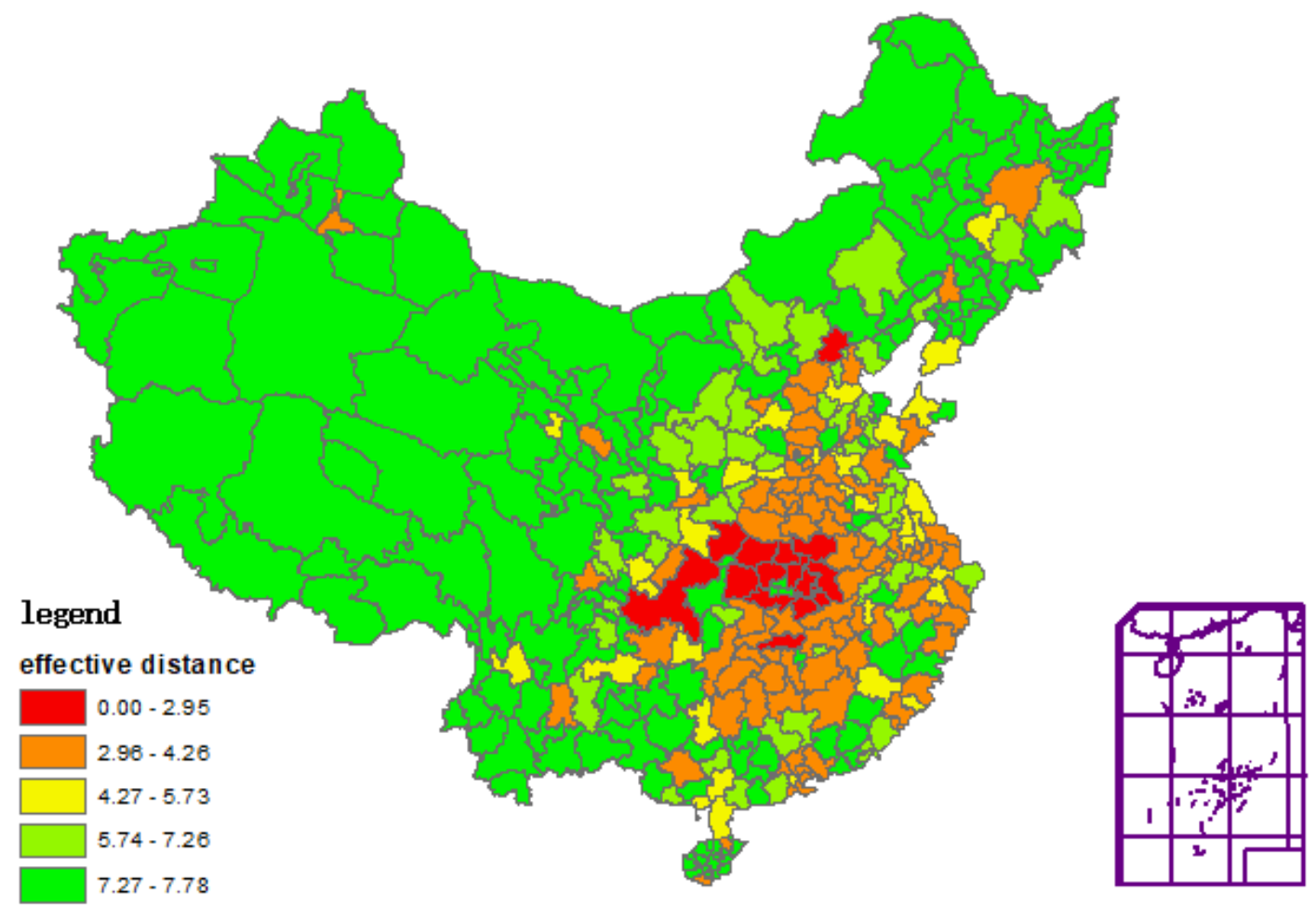

516 Figure 6 Effective Distance between Wuhan and Each Other Prefectural-level Administrative

517 Regions on February 19, 2020

\section{$518 \quad 4.4$ Statistical Description}

519 The data samples in this paper is composed of balance panel data of 279 prefecture-level 520 administrative regions from January 1 to February 19, 2020, and the descriptive statistics of 521 related variables are shown as table 4.

\begin{tabular}{|c|c|c|c|c|c|c|c|}
\hline Variables & Implication & Notation & $\mathbf{N}$ & mean & sd & $\min$ & $\max$ \\
\hline Explained & Increased rate of & Rate & 13,950 & 0.0851 & 0.443 & 0 & 19 \\
\hline
\end{tabular}




\begin{tabular}{|c|c|c|c|c|c|c|c|}
\hline Explanatory & average temperature & AT & 13,950 & 3.789 & 9.0667 & -31.2 & 26.4 \\
\hline \multirow[t]{5}{*}{ variable } & Relative humidity & $\mathrm{RH}$ & 13,850 & 71.17 & 17.15 & 6 & 102 \\
\hline & Public health measures & Measure & 13,950 & 3.729 & 3.912 & 0 & 10 \\
\hline & score & & & & & & \\
\hline & Number of registered & Pop & 13,950 & 171.3 & 226.3 & 16 & 2,451 \\
\hline & population & & & & & & \\
\hline \multirow{4}{*}{ Control variable } & Number of hospital & Hospital_bed & 13,950 & 12,906 & 17,135 & 920 & 142,708 \\
\hline & beds & & & & & & \\
\hline & GDP per capita & Per GDP & 13,950 & 92,348 & 379,890 & 17,890 & $6.400 \mathrm{e}+06$ \\
\hline & effective distance & Distance & 13,950 & 5.722 & 1.874 & 0 & 7.785 \\
\hline
\end{tabular}

523

524 5. Results

$525 \quad 5.1$ Does Weather Condition Matter?

$526 \quad$ 5.1.1 Baseline regression

527 Table 5 reports the results of the baseline regression. The explanatory variable is the growth rate

528 of cumulative cases, and the explanatory variables are average temperature and relative humidity.

529 Column (1) only introduces the explanatory variables, and column (2) - column (5) adds the control

530 variables in sequence based on column (1). The results show that the average temperature

531 coefficient is significantly negative, which indicates that there is a significant negative casual

532 relationship between temperature and the growth rate of the confirmed cases. In cities with higher

533 temperatures, the transmission rate of the epidemic is slow, and the growth rate of confirmed cases

534 is lower; on the contrary, the virus transmission ability is stronger in cold conditions. Similarly,

535 the coefficient of relative humidity is significantly negative, which indicates that there is a 
536 significant negative casual relationship between the relative humidity and the growth rate of 537 confirmed cases. In cities with higher humidity, the growth rate of confirmed cases is lower, while 538 in cities with low humidity, the epidemic spread rate is faster, and the growth rate of confirmed 539 cases is higher.

\begin{tabular}{|c|c|c|c|c|c|}
\hline & (1) & (2) & (3) & (4) & (5) \\
\hline & rate & rate & rate & rate & rate \\
\hline \multirow[t]{2}{*}{ AT } & $-0.0036 * * *$ & $-0.0032 * * *$ & $-0.0033 * * *$ & $-0.0033 * * *$ & $-0.0036 * * *$ \\
\hline & $(0.0011)$ & $(0.0011)$ & $(0.0011)$ & $(0.0011)$ & $(0.0011)$ \\
\hline \multirow[t]{2}{*}{ RH } & $-0.0017 * * *$ & $-0.0015 * * *$ & $-0.0015^{* * *}$ & $-0.0015^{* * *}$ & $-0.0015 * * *$ \\
\hline & $(0.0003)$ & $(0.0003)$ & $(0.0003)$ & $(0.0003)$ & $(0.0003)$ \\
\hline \multirow[t]{2}{*}{ Measure } & & $-0.0220 * * *$ & $-0.0222 * * *$ & $-0.0220 * * *$ & $-0.0223 * * *$ \\
\hline & & $(0.0030)$ & $(0.0030)$ & $(0.0030)$ & $(0.0030)$ \\
\hline \multirow[t]{2}{*}{ Pop } & & & $0.0299 * * *$ & $0.0415 * * *$ & $0.0316 * * *$ \\
\hline & & & $(0.0065)$ & (0.0109) & $(0.0112)$ \\
\hline \multirow[t]{2}{*}{ Hospital } & & & & -0.0085 & $-0.0132 * *$ \\
\hline & & & & $(0.0064)$ & $(0.0065)$ \\
\hline \multirow[t]{2}{*}{ Distance } & & & & & $-0.0133 * * *$ \\
\hline & & & & & $(0.0038)$ \\
\hline Constant & $0.0709 * * *$ & 0.0162 & $-0.1226 * * *$ & $-0.1007 * *$ & 0.0663 \\
\hline
\end{tabular}


$(0.0262)$

12,555

0.033

YES

Time Trend

Province FE

Time FE
(0.0271)

12,555

0.038

YES

YES

YES
(0.0407)

12,555

0.039

YES

YES

YES
(0.0438)

(0.0651)

12,555

12,555

0.039

0.040

YES

YES YES

YES

YES

542 We also analyze control variables. It is concluded that the coefficient of public health measures is

543 significantly negative, indicating that taking public health measures is essential to mitigate the 544 epidemic. The better the public health measures are, the lower the growth rate of the number of 545 confirmed cases is. The coefficient of population size is significantly positive. The vast population 546 size will increase the difficulty of isolating person-to-person contact which has an adverse effect 547 on blocking the further spread of infectious diseases. The coefficient of both the number of beds 548 in health institutions and effective distance are significantly negative. The number of beds in health 549 institutions represents the medical resources condition of the city, and cities with richer medical 550 resources are more capable of mitigating the deterioration of the epidemic. The shorter the 551 effective distance to Wuhan, the more severe the outbreak of the epidemic, which is in line with 552 theoretical expectations.

\section{$553 \quad$ 5.1.2 Nonlinear relationship or conditional linear relationship?}

554 We have preliminarily verified that the average temperature and relative humidity negatively 555 affects the epidemic, however, we're also concerned that whether this negative relationship only 556 holds up over a certain interval, or whether there is the possibility of a nonlinear relationship. 
557 Therefore, we try to introduce the quadratic terms of the average temperature and relative humidity 558 respectively, to further explore the influence of weather conditions on the epidemic.

\begin{tabular}{|c|c|c|}
\hline & rate & rate \\
\hline \multirow[t]{2}{*}{$\overline{\mathrm{AT}}$} & $-0.0030 * * *$ & $-0.0034 * * *$ \\
\hline & $(0.0011)$ & $(0.0011)$ \\
\hline \multirow[t]{2}{*}{$\mathrm{AT}^{*} \mathrm{AT}$} & $-0.0002 * * *$ & \\
\hline & $(0.0001)$ & \\
\hline \multirow[t]{2}{*}{ RH } & $-0.0015 * * *$ & $0.0032 *$ \\
\hline & $(0.0003)$ & $(0.0019)$ \\
\hline \multirow[t]{2}{*}{$\mathrm{RH}^{*} \mathrm{RH}$} & & $-0.00003 * * *$ \\
\hline & & $(0.0000)$ \\
\hline \multirow[t]{2}{*}{ Measure } & $-0.0216 * * *$ & $-0.0223 * * *$ \\
\hline & $(0.0030)$ & $(0.0030)$ \\
\hline \multirow[t]{2}{*}{ Pop } & $0.0304 * * *$ & $0.0313 * * *$ \\
\hline & $(0.0112)$ & $(0.0112)$ \\
\hline Hospital & $-0.0126^{*}$ & $-0.0138 * *$ \\
\hline
\end{tabular}


(0.0065)

Distance

$-0.0130 * * *$

(0.0038)

Constant

0.0832

(0.0652)

12,555

0.041

YES

YES

YES

$-7$
$(0.0065)$

$-0.0135 * * *$

(0.0038)

$-0.0840$

(0.0874)

12,555

0.041

YES

YES

YES

46

561 Table 6 reports the regression results of the nonlinear model and figure 7 shows the nonlinear

562 relationship between the average temperature, relative humidity and the epidemic. We find that

563 although the coefficients of quadratic terms of both average temperature and relative humidity are

564 significant, it can be seen from the figure 7 that the positive correlation is almost insignificant on

565 the left side of the inflection point. In order to further confirm whether the negative relationship is

566 within a certain value range of explanatory variables, we perform sub-sample regression based on

567 the value of the inflection point (-b/2a), the inflection point of the average temperature is $-7^{\circ} \mathrm{C}$, and 568 the inflection point of the relative humidity is $46 \%$. 

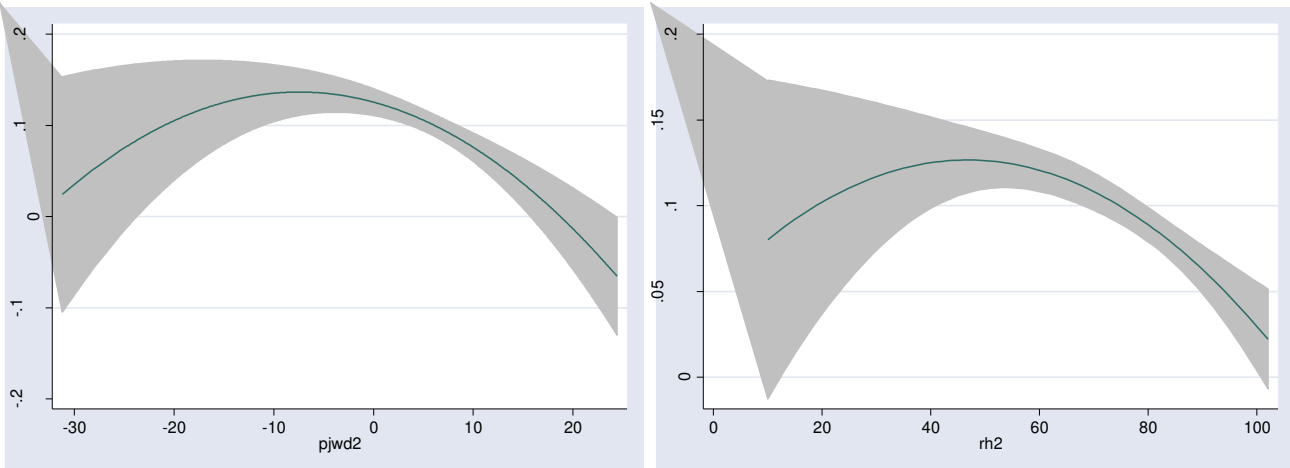

Figure 7 Whether There is a Nonlinear Relationship.

571 Table 7 reports the results of the sub-sample regression. It can be seen that when the average 572 temperature is more than $-7^{\circ} \mathrm{C}$, it has a negative correlation with the growth rate of the cases; when

573 it is lower than $-7{ }^{\circ} \mathrm{C}$, then there is no significant correlation between the average temperature and 574 the epidemic; Similarly, when the relative humidity is higher than $46 \%$, there is a negative 575 correlation between it and the cumulative case growth rate, but when it is lower than 46, the 576 decrease of relative humidity will not affect the epidemic. Therefore, there is a conditional linear 577 relationship between weather conditions and the COVID-19 epidemic.

Table 7. Conditional Negative Linear Relationship

$\mathrm{AT}<-7^{\circ} \mathrm{C} \quad \mathrm{AT}>=-7^{\circ} \mathrm{C} \quad \mathrm{RH}<46 \% \quad \mathrm{RH}>=46 \%$

AT

$-0.0011$

$-0.0049 * * *$

$-0.0003$

$-0.0033 * * *$

(0.0014)

(0.0013)

(0.0021)

(0.0012)

RH

$-0.0005$

$-0.0018 * * *$

$-0.0002$

$-0.0019 * * *$ 
$(0.0006)$

Constant

Observations

R-squared

Control Variables

Time Trend

Province FE

Time FE
0.0041
YES

YES
(0.0004)

0.1015

$(0.0733)$

11,014

0.043

YES

YES

YES

YES
(0.0006)

0.1487

0.026

YES

YES
YES

YES
$(0.0720)$

11,468

0.044

YES

YES

$(0.0004)$

0.0775

YES

YES

\subsubsection{Robustness}

581 The purpose of robustness check is to demonstrate whether the results change with the adjustment

582 of parameter setting. If the results show that the signs or significance changes after the parameter

583 setting is adjuested, it indicates that the results are not robust and the problem needs to be found

584 out. There are generally three methods of robustness check: 1) In terms of data, adjust the

585 classification according to different standards, and check whether the results are still significant.

586 2) In terms of variables, replace the orign variables with other proxy. For example, the average

587 temperature can be changed to the maximum temperature. 3) In terms of econometrics 588 identification method, try a variety of identification strategies, such as ordinary least square (OLS),

589 limited information maximum likelihood (LIML), and generalized method of moments (GMM). 
590 First, considering that the epidemic first broke out in city of Wuhan and had a severe impact on 591 other cities in Hubei Province, we eliminate the sample of Hubei Province; Second, we adjust the 592 hypothesis about the length of the incubation period, assuming that the incubation period is 6 days 593 and 7 days respectively, and then perform the regression again. The results of the robustness are 594 reported in Table 8. It can be seen that the sign and significance of the coefficients are consistent 595 with the baseline regression, indicating that the conclusion is still robust after changing the sample 596 selection basic assumptions.

Table 8 Robustness

\section{Panel A without Hubei Province}

\begin{tabular}{|c|c|c|c|c|}
\hline & (1) & (2) & (3) & (4) \\
\hline & & ${ }^{\circ} \mathrm{C}$ & $\mathrm{RH}$ & $6 \%$ \\
\hline AT & $-0.0048 * * *$ & $-0.0048 * * *$ & $-0.0029 * * *$ & $-0.0031 * * *$ \\
\hline & $(0.0010)$ & $(0.0010)$ & $(0.0010)$ & $(0.0010)$ \\
\hline RH & $-0.0017 * * *$ & $-0.0015 * * *$ & $-0.0018 * * *$ & $-0.0016^{* * *}$ \\
\hline & $(0.0003)$ & $(0.0003)$ & $(0.0003)$ & $(0.0003)$ \\
\hline Observations & 10,474 & 10,474 & 10,845 & 10,845 \\
\hline R-squared & 0.042 & 0.052 & 0.041 & 0.053 \\
\hline Control Variables & No & YES & NO & YES \\
\hline Time Trend & YES & YES & YES & YES \\
\hline Province FE & YES & YES & YES & YES \\
\hline Time FE & YES & YES & YES & YES \\
\hline & (1) & (2) & (3) & (4) \\
\hline
\end{tabular}




\begin{tabular}{|c|c|c|c|c|}
\hline \multicolumn{5}{|c|}{ Panel B Adjust the incubation period } \\
\hline & The & The & The & The \\
\hline & incubation & incubation & incubation & incubation \\
\hline & period is 6 & period is 7 & period is 6 & period is 7 \\
\hline & days & days & days & days \\
\hline & \multicolumn{2}{|c|}{$\mathrm{AT}>=-7^{\circ} \mathrm{C}$} & \multicolumn{2}{|c|}{$\mathrm{RH}>=46 \%$} \\
\hline AT & $-0.0057 * * *$ & $-0.0065^{* * *}$ & $-0.0036^{* * *}$ & $-0.0047 * * *$ \\
\hline & $(0.0014)$ & $(0.0014)$ & $(0.0013)$ & $(0.0013)$ \\
\hline \multirow[t]{2}{*}{ RH } & $-0.0018 * * *$ & $-0.0009 * *$ & $-0.0020 * * *$ & $-0.0011 * * *$ \\
\hline & $(0.0004)$ & $(0.0004)$ & $(0.0004)$ & $(0.0004)$ \\
\hline Observations & 10,757 & 10,510 & 11,101 & 10,826 \\
\hline R-squared & 0.043 & 0.041 & 0.044 & 0.042 \\
\hline Control Variables & YES & YES & YES & YES \\
\hline Time Trend & YES & YES & YES & YES \\
\hline Province FE & YES & YES & YES & YES \\
\hline Time FE & YES & YES & YES & YES \\
\hline
\end{tabular}

\section{$599 \quad$ 5.1.4 Endogenity Treatment}

600 Since the spread of the epidemic may be affected by some unobservable factors, the problem of 601 omitted variables may not be avoided in the regression. In this case, the influence of the omitted 602 variables is included in the error term where when it is related to other explanatory variables, the 603 endogenous problems arise. In order to address the endogenous problems caused by omitted 604 variables, we use the instrumental variable approach to re-estimate. The selection of instrument 
605 variables needs to meet two requirements: the first is that there is a significant relationship between

606 the instrumental variables and the endogenous explanatory variables; and the second is that the

607 instrument variable must be exogenous. We take the 1st lag of the explanatory variable as an

608 instrument variable, and in order to ensure the robustness of the results, we respectively

609 use two-stage least squares (2SLS), limited information maximum likelihood (LIML), and

610 generalized method of moments (GMM). Table 9 reports the results of the instrumental variable

611 approach. We can say that there is no obvious change in the sign and significance of the

612 coefficients. The results of the instrumental variables approach are consistent with the baseline

613 regression, indicating that the conclusion is still robust after considering endogeneity.

\begin{tabular}{|c|c|c|c|c|c|c|}
\hline & (1) & (2) & (3) & (4) & (5) & (6) \\
\hline & \multicolumn{3}{|c|}{$\mathrm{AT}>=-7^{\circ} \mathrm{C}$} & \multicolumn{3}{|c|}{$\mathrm{RH}>=46 \%$} \\
\hline & 2SLS & LIML & GMM & 2SLS & LIML & GMM \\
\hline \multirow[t]{3}{*}{$\mathrm{AT}$} & $-0.0062 * * *$ & $-0.0062 * * *$ & $-0.0062 * * *$ & $-0.0033 * * *$ & $-0.0033 * * *$ & - \\
\hline & & & & & & $0.0033 * * *$ \\
\hline & $(0.0010)$ & $(0.0010)$ & $(0.0010)$ & $(0.0008)$ & $(0.0008)$ & $(0.0008)$ \\
\hline \multirow[t]{3}{*}{ RH } & $-0.0019 * * *$ & $-0.0019 * * *$ & $-0.0019 * * *$ & $-0.0032 * * *$ & $-0.0032 * * *$ & - \\
\hline & & & & & & $0.0032 * * *$ \\
\hline & $(0.0004)$ & $(0.0004)$ & $(0.0004)$ & $(0.0007)$ & $(0.0007)$ & $(0.0007)$ \\
\hline Observations & 10,732 & 10,732 & 10,732 & 11,101 & 11,101 & 11,101 \\
\hline R-squared & 0.042 & 0.042 & 0.042 & 0.042 & 0.042 & 0.042 \\
\hline
\end{tabular}




$\begin{array}{lcccccc}\text { Control } & \text { YES } & \text { YES } & \text { YES } & \text { YES } & \text { YES } & \text { YES } \\ \text { Variables } & & & & & \\ \text { Time Trend } & \text { YES } & \text { YES } & \text { YES } & \text { YES } & \text { YES } & \text { YES } \\ \text { Province FE } & \text { YES } & \text { YES } & \text { YES } & \text { YES } & \text { YES } & \text { YES } \\ \text { Time FE } & \text { YES } & \text { YES } & \text { YES } & \text { YES } & \text { YES } & \text { YES }\end{array}$

\section{$616 \quad 5.2$ Further Exploration}

\section{$617 \quad$ 5.2.1 Where is more dangerous?}

618 Since we have proved that when the average temperature is more significant than $-7^{\circ} \mathrm{C}$, the average

619 temperature is negatively correlated with the spread of the epidemic, and when the relative 620 humidity is greater than $46 \%$, the relative humidity is negatively correlated with the epidemic, then 621 it can be inferred that when the temperature is $-7^{\circ} \mathrm{C}$, the spread of the epidemic is the most serious; 622 when the relative humidity is $46 \%$, the spread of the epidemic is the most serious. So, in China, a 623 county with vast territory and wide difference in climatic conditions, which cities have more 624 favorable climate conditions for the development of the epidemic?

625 We make statistics of the prefecture-level administrative regions which meet the requirements that 626 the average temperature is $-7^{\circ} \mathrm{C} \pm$ one standard deviation $\left(9.0667^{\circ} \mathrm{C}\right)$, the relative humidity is $46 \%$ $627 \pm$ one standard deviation (17.15\%), and both meet the two conditions simultaneously from January 6281,2020 to February 19. We construct a dummy variable of whether the city falls into the interval 629 for regression and count the number of days that each city meets the conditions. Table 10 reports 630 the impact of the dangerous weather on the epidemic. It can be seen that the epidemic is indeed 631 more severe in the dangerous weather. 


\begin{tabular}{|c|c|c|}
\hline & $\begin{array}{l}\text { (1) } \\
\text { rate }\end{array}$ & $\begin{array}{l}\text { (2) } \\
\text { rate }\end{array}$ \\
\hline $\begin{array}{l}\text { City whith AT - } \\
7^{\circ} \mathrm{C} \pm 9.0667^{\circ} \mathrm{C}\end{array}$ & $0.0472 * * *$ & \\
\hline $\begin{array}{l}\text { City with RH } \\
46 \% \pm 17.15 \%\end{array}$ & & $(0.0110)$ \\
\hline Observations & 12,555 & 12,555 \\
\hline R-squared & 0.039 & 0.038 \\
\hline Control Variables & YES & YES \\
\hline Time Trend & YES & YES \\
\hline Province FE & YES & YES \\
\hline Time FE & YES & YES \\
\hline
\end{tabular}

634 Table 11, Table 12, and Table 13 report the top dangerous areas in the country under three 635 conditions. It can be seen from the results that from a weather perspective, the winter in cities 636 such as Chifeng and Zhangjiakou is more conducive to the outbreak of the epidemic.

\begin{tabular}{|c|c|}
\hline City & number of days \\
\hline Chengde & 45 \\
\hline
\end{tabular}




\begin{tabular}{|c|c|}
\hline Hohhot & 45 \\
\hline Wuhai & 45 \\
\hline Bayannur & 45 \\
\hline LanZhou & 45 \\
\hline Baiyin & 45 \\
\hline Zhangye & 45 \\
\hline Dingxi & 45 \\
\hline Xining & 45 \\
\hline Shizuishan & 45 \\
\hline Urumqi & 45 \\
\hline
\end{tabular}

Table 12 the Number of Days Complying with Dangerous Relative Humidity

\begin{tabular}{|c|c|c|}
\hline Rank & City & number of days \\
\hline 1 & Chifeng & 47 \\
\hline 2 & Zhangjiakou & 46 \\
\hline 3 & Longnan & 44 \\
\hline 4 & Ulanqab & 42 \\
\hline 5 & Jinzhou & 42 \\
\hline 6 & Lijiang & 40 \\
\hline 7 & Chaoyang & 40 \\
\hline 8 & Chengde & 38 \\
\hline 9 & Zhangye & \\
\hline
\end{tabular}




\begin{tabular}{|c|c|c|}
\hline 10 & Baoshan & 37 \\
\hline 11 & Lincang & 37 \\
\hline 12 & Panzhihua & 37 \\
\hline
\end{tabular}

640

Table 13 the Number of Days that Meet Both the Two Conditions

\begin{tabular}{|c|c|c|}
\hline Rank & City & number of days \\
\hline 1 & Zhangjiakou & 42 \\
\hline 2 & Chifeng & 39 \\
\hline 3 & Ulanqab & 36 \\
\hline 4 & Chengde & 35 \\
\hline 5 & Jinzhou & 33 \\
\hline 6 & Zhangye & 33 \\
\hline 7 & Xinzhou & 31 \\
\hline 8 & Chaoyang & 31 \\
\hline 9 & Liaoyang & \\
\hline 10 & Anshan & \\
\hline
\end{tabular}

642

$643 \quad$ 5.2.2 What is the difference between heterogeneous regions?

644 The difference in weather conditions among different regions in China is affected not only by the

645 large latitude crossing between north and south and also by the significant difference in altitude 646 and the location of coastal and inland. For this reason, we carry out sub-sample regression 647 according to geographical location. 
648 The results are shown in Table 14. Panel A in Table 14 reports the results according to the samples 649 in the east, middle, and west. The eastern region includes Beijing, Tianjin, Hebei, Liaoning, 650 Shanghai, Jiangsu, Zhejiang, Fujian, Shandong, Guangdong, and Hainan. The middle region 651 includes Shanxi, Inner Mongolia, Jilin, Heilongjiang, Anhui, Jiangxi, Henan, Hubei, Hunan and 652 Guangxi; the western region includes Sichuan, Guizhou, Yunnan, Tibet, Shanxi, Gansu, Qinghai, 653 Ningxia, and Xinjiang. ${ }^{8}$ It can be seen that the coefficient of average temperature is still 654 significantly negative in the eastern and western regions, in which the influence of average 655 temperature in the east is greater than that in the west, but it doesn't work in the middle. The effect 656 of relative humidity is the most significant in the middle, followed by the east and the weakest in 657 the west. Panel B reports the sub-sample results of the coastal and inland areas. According to the 658 China Marine Statistical Yearbook, coastal areas are defined as areas with coastlines, which are 659 divided into coastal provinces, autonomous regions and municipalities. At present, there are 53 660 coastal cities and 242 coastal counties. It shows that the influence of both average temperature and 661 relative humidity is greater in the coastal areas, and the role of weather conditions is more 662 important in the coastal areas than inland.

Panel A East Middle and West

(1)

\begin{tabular}{|c|c|c|c|c|c|c|}
\hline & & $\mathrm{AT}>=-7^{\circ} \mathrm{C}$ & & & $\mathrm{RH}>=46 \%$ & \\
\hline & East & Middle & West & East & Middle & West \\
\hline AT & $-0.0059 * * *$ & -0.0025 & $-0.0056 * * *$ & $-0.0045 * *$ & 0.0000 & $-0.0048 * * *$ \\
\hline & $(0.0020)$ & $(0.0035)$ & $(0.0015)$ & $(0.0020)$ & $(0.0026)$ & $(0.0014)$ \\
\hline
\end{tabular}

\footnotetext{
${ }^{8}$ According to the classification of the National Bureau of Statistics of China, http://www.stats.gov.cn/.
} 


\begin{tabular}{lcccccc} 
RH & $-0.0019 * * *$ & $-0.0027 * * *$ & $-0.0013 * * *$ & $-0.0021 * * *$ & $-0.0025^{* * *}$ & $-0.0015^{* * *}$ \\
& $(0.0006)$ & $(0.0009)$ & $(0.0004)$ & $(0.0006)$ & $(0.0008)$ & $(0.0004)$ \\
Observations & 4,128 & 3,604 & 3,257 & 4,082 & 4,255 & 3,040 \\
R-squared & 0.054 & 0.041 & 0.040 & 0.054 & 0.041 & 0.047 \\
Control Variables & YES & YES & YES & YES & YES & YES \\
Time Trend & YES & YES & YES & YES & YES & YES \\
Province FE & YES & YES & YES & YES & YES & YES \\
Time FE & YES & YES & YES & YES & YES & YES \\
\hline
\end{tabular}

Panel B Coastal and Inland
(1)
(2)
(3)
(4)

Coastal Inland Coastal Inland

\begin{tabular}{lcccc} 
& \multicolumn{2}{c}{$\mathrm{AT}>=-7^{\circ} \mathrm{C}$} & \multicolumn{2}{c}{$\mathrm{RH}>=46 \%$} \\
\hline AT & $-0.0098^{* *}$ & $-0.0044^{* * *}$ & $-0.0101^{* *}$ & $-0.0025^{*}$ \\
& $(0.0040)$ & $(0.0015)$ & $(0.0042)$ & $(0.0013)$ \\
RH & $-0.0018^{*}$ & $-0.0017^{* * *}$ & $-0.0021^{*}$ & $-0.0017 * * *$ \\
& $(0.0010)$ & $(0.0004)$ & $(0.0011)$ & $(0.0004)$ \\
Observations & 2,191 & 8,798 & 2,101 & 9,276 \\
R-squared & 0.051 & 0.043 & 0.051 & 0.044 \\
Control Variables & YES & YES & YES & YES \\
Time Trend & YES & YES & YES & YES \\
Province FE & YES & YES & YES & YES \\
Time FE & YES & YES & YES & YES \\
\hline
\end{tabular}


666 Both Polgreen \& Polgreen (2018) and Paraskevis et al. (2020) point out that studying the impact

667 of weather conditions on the spread of the epidemic cannot separate public health interventions

668 and human behavior patterns. In order to further analyze the effects of other important factors,

669 we introduce the interation term of the explanatory variables with diurnal temperature variation ,

670 public health measures, and social public opinion to explore the moderating effects of these

671 factors on weather conditions affecting the epidemic.

672 Jaagus et al. (2014) believe that the main influencing factors of the temperature difference between

673 day and night are latitude, altitude, and location of the land and sea. A large temperature difference

674 between day and night weakens the immune system and makes people more susceptible to

675 infection under equal conditions. The temperature difference between day and night (TD) is the

676 difference between the highest temperature (HT) and the lowest temperature (LT) each day. The

677 calculation method is as follows.

678

$$
\text { TD }=\text { HT-LT }
$$

679 The temperature difference between day and night in China on February 19, 2020 is shown in 680 figure 8. 


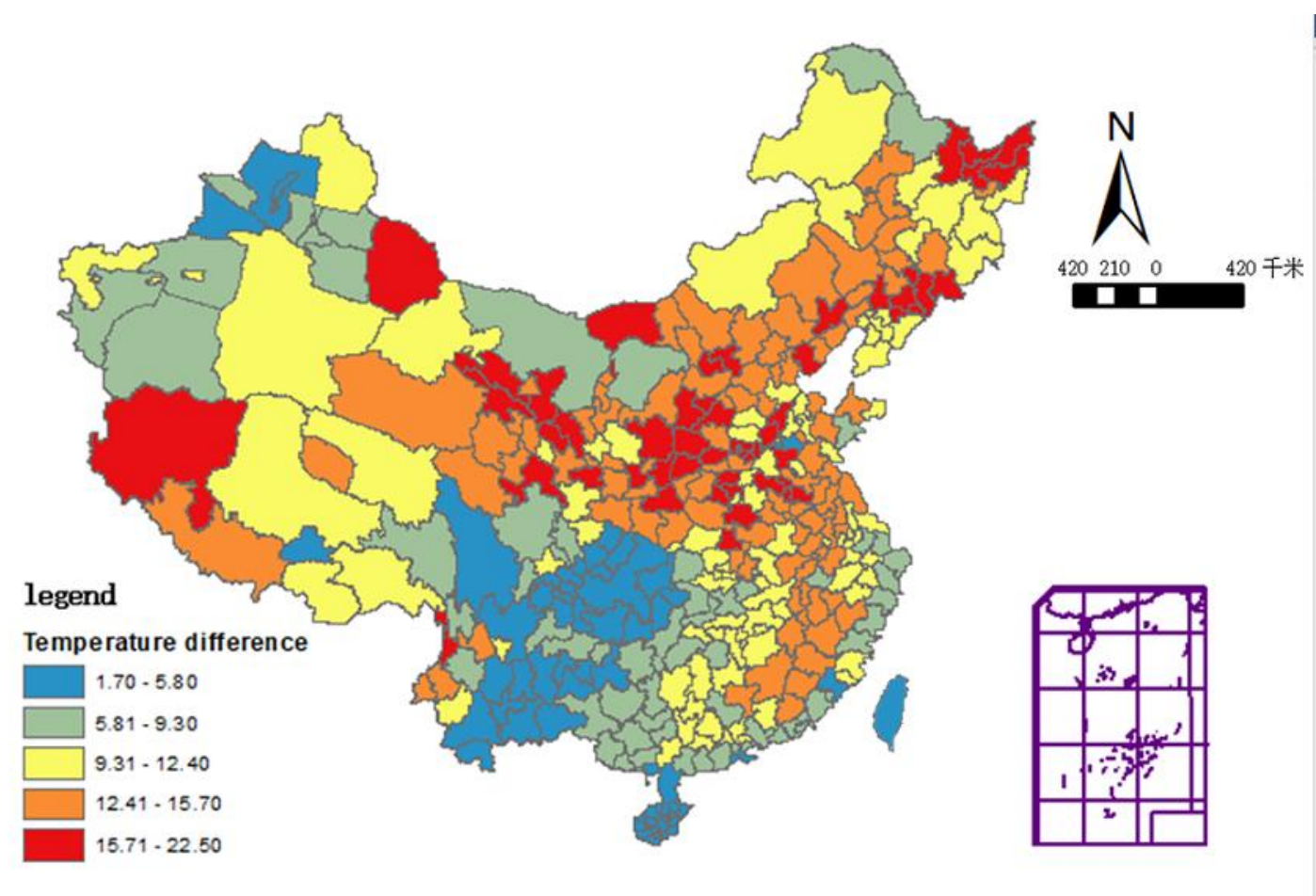

Figure 8 The temperature Difference between Day and Night in China on February 19, 2020

684 Rapid and strict public health measures can effectively prevent the further spread of the epidemic, 685 and good public opinion can enhance the public's attention to the epidemic to improve the 686 awareness of prevention. Theoretically, both the factors are conducive to reducing the impact of 687 weather conditions on the epidemic. The data of social public opinion is based on the search service 688 provided by Baidu Index. We select six epidemic-related terms: the COVID-19, pneumonia, Zhong 689 Nanshan, pneumonia symptoms, mask, and correct wearing of masks to reflect the public's 690 response to the epidemic, apply big data mining technology to collect data, and add up to obtain 691 daily data. Social public opinion in China on February 19, 2020 is shown in figure 9. 


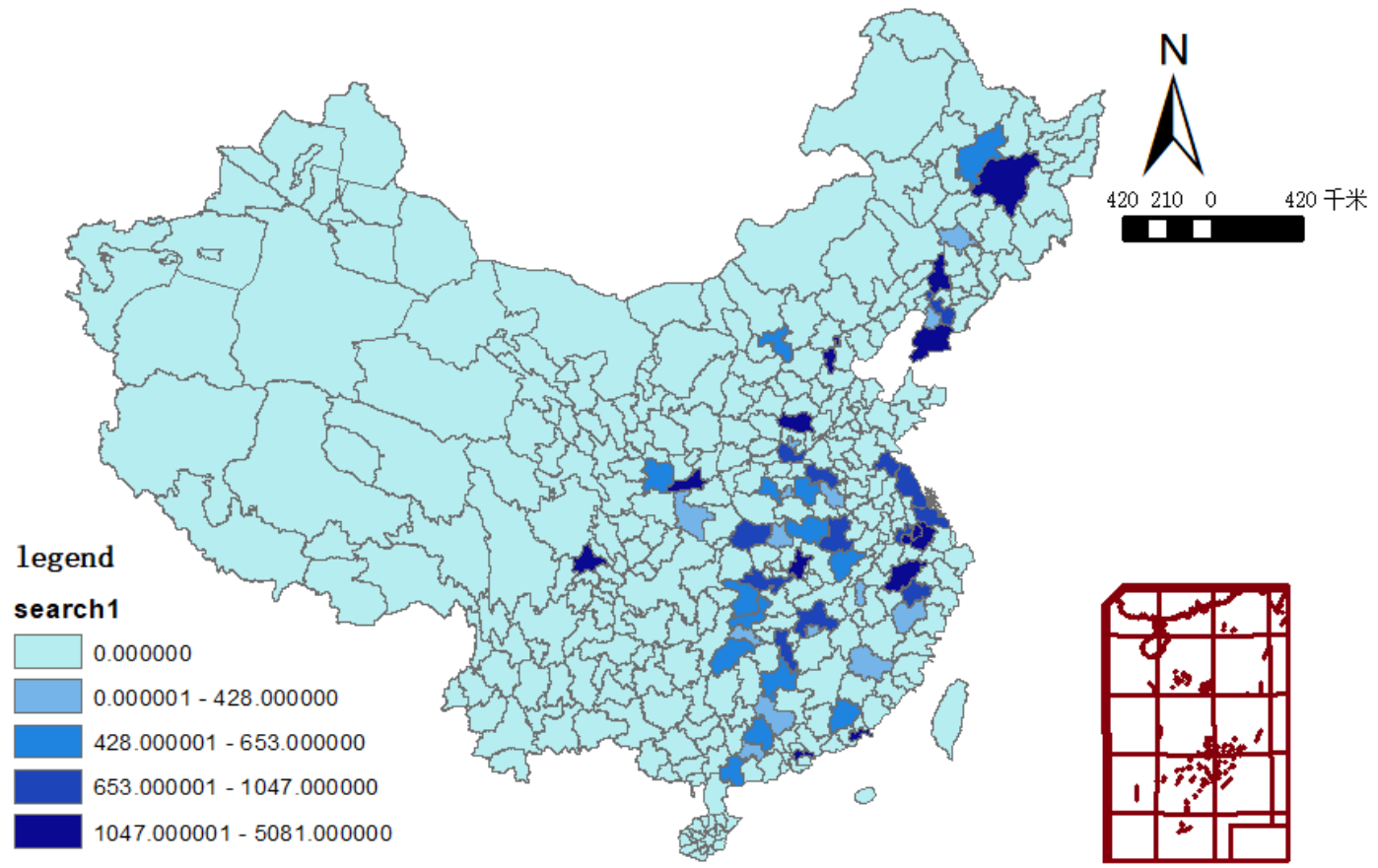

694 We divide the three variables of diurnal temperature difference, public health measures, and social 695 public opinion into high, medium, and low, respectively, generate dummy variables, and construct 696 the interaction terms between dummy variables and the weather condition variables. The results 697 are shown in Table 15. Column (1) introduces the interaction between average temperature and 698 high diurnal temperature variation (hightf), and column (2) introduces the interaction between 699 relative humidity and the item; similarly, column (3) and column (4) presents the results of public 700 health measures, and column (3) and column (4) presents the results of social publicopinion.

701 It can be seen that, whether it is average temperature or relative humidity, the coefficient of the 702 interaction with high diurnal temperature difference (hightf) is significantly negative, that is, the 703 increase in diurnal temperature differences lead to a stronger impact of weather conditions on the 704 increase in the rate of confirmed COVID-19 cases, especially in dry and cold regions, where higher 705 diurnal temperature differences will increase the risk of the spread of the epidemic. On the contrary, 
706 the coefficients of the interaction with high public health measures (highpolicy) and high social 707 public opinion (highopinion) are both significantly positive, indicating that the improvement of 708 public health measures and social public opinion can weaken the influence of average temperature 709 and relative humidity on the COVID - 19 confirmed cases growth rate. It can be concluded that 710 strict public health measures and sound public opinion can mitigate the adverse effects of cold and 711 dry weather on the spread of the epidemic, which reinforces the importance of public health 712 measures and attention to public response.

Table 15 Exploration of Moderating Effects

\begin{tabular}{|c|c|c|c|c|c|c|}
\hline & (1) & (2) & (3) & (4) & (5) & (6) \\
\hline & rate & rate & rate & rate & rate & rate \\
\hline \multirow[t]{2}{*}{ AT } & $-0.0033 * *$ & $-0.0033 * * *$ & $-0.0063 * * *$ & $-0.0027 * *$ & $-0.0056 * * *$ & $-0.0032 * * *$ \\
\hline & $(0.0015)$ & $(0.0012)$ & $(0.0014)$ & $(0.0012)$ & $(0.0014)$ & $(0.0012)$ \\
\hline \multirow[t]{2}{*}{ RH } & $-0.0020 * * *$ & $-0.0018 * * *$ & $-0.0021 * * *$ & $-0.0034 * * *$ & $-0.0020 * * *$ & $-0.0022 * * *$ \\
\hline & $(0.0004)$ & $(0.0004)$ & $(0.0004)$ & $(0.0004)$ & $(0.0004)$ & $(0.0004)$ \\
\hline \multirow[t]{2}{*}{ AT*hightf } & $-0.0034 * * *$ & & & & & \\
\hline & $(0.0011)$ & & & & & \\
\hline \multirow[t]{2}{*}{$\mathrm{RH}^{*}$ hightf } & & $-0.0005 * * *$ & & & & \\
\hline & & $(0.0001)$ & & & & \\
\hline \multirow[t]{2}{*}{ AT*highpolicy } & & & $0.0040 * * *$ & & & \\
\hline & & & $(0.0013)$ & & & \\
\hline \multirow[t]{2}{*}{ RH* highpolicy } & & & & $0.0028 * * *$ & & \\
\hline & & & & $(0.0002)$ & & \\
\hline \multirow[t]{2}{*}{ AT*highopinion } & & & & & $0.0030 * * *$ & \\
\hline & & & & & $(0.0011)$ & \\
\hline \multirow[t]{2}{*}{ RH*highopinion } & & & & & & $0.0004 * * *$ \\
\hline & & & & & & $(0.0001)$ \\
\hline Observations & 10,989 & 11,377 & 10,989 & 11,377 & 10,989 & 11,377 \\
\hline R-squared & 0.038 & 0.037 & 0.038 & 0.050 & 0.037 & 0.036 \\
\hline
\end{tabular}




\begin{tabular}{|c|c|c|c|c|c|c|}
\hline Control Variables & YES & YES & YES & YES & YES & YES \\
\hline Time Trend & YES & YES & YES & YES & YES & YES \\
\hline Province FE & YES & YES & YES & YES & YES & YES \\
\hline Time FE & YES & YES & YES & YES & YES & YES \\
\hline
\end{tabular}

\section{6. Conclusion and Discussion}

716 In this paper, we collect the COVID-19 related prefecture-daily data of mainland China from

717 January 1, 2020, to February 19, calculate indicators such as growth rate of the confirmed cases, 718 average temperature, relative humidity, the score of public health measures, and effective distance, 719 and empirically test the influence of weather conditions on the COVID-19 epidemic applying two720 way fixed effect model of multiple linear regression. The main conclusions are as follows.

722 First, we analyze the effects of the average temperature on the growth rate of the confirmed cases, 723 and we find that there is a conditional negative linear relationship between the weather conditions 724 and the epidemic. When the average temperature is greater than $-7^{\circ} \mathrm{C}$, there is a significant negative 725 causal relationship between the average temperature and the growth rate in the confirmed cases, 726 while when the average temperature is less than $-7^{\circ} \mathrm{C}$, it has no significant effect on the epidemic.

727 Similarly, when relative humidity is greater than $46 \%$, it has a negative impact on the spread of 728 the epidemic, while when relative humidity is less than $46 \%$, the reduction inrelative humidity will 729 no longer affect the epidemic. In robustness checks, we try to remove data of Hubei province from 730 the whole sample, which is most affected by the epidemic in China, and to adjust the assumption 731 of incubation period length in the calculation of actual cumulative case growth rate; considering 732 the possible endogeneity, we take the first-order lag of the main explanatory variables as an 
733 instrument variable, and to ensure the robustness of the results, we respectively use two-stage least

734 squares (2SLS), limited information maximum likelihood (LIML), and generalized method of 735 moments (GMM) to reestimate the coefficient.

736 Second, according to the conditional negative causality between weather conditions and the 737 COVID-19 epidemic, we make statistics of the prefecture-level administrative regions which meet 738 the requirements that the average temperature is $-7^{\circ} \mathrm{C} \pm$ one standard deviation $\left(9.0667^{\circ} \mathrm{C}\right)$, the 739 relative humidity is $46 \% \pm$ one standard deviation $(17.15 \%)$, and both meet the two conditions 740 simultaneously from January 1, 2020, to February 19. It is concluded that from the perspective of 741 weather conditions, prefecture-level administrative regions such Chifeng, Zhangjiakou, and 742 Ulanqab are more conducive to the outbreak of the epidemic in winter.

743 Third, we explore the heterogeneity of the influence of weather conditions. Based on the 744 geographical characteristics of China, we conduct sample regression according to the eastern, 745 middle, west, and inland, coastal regions, respectively. We find that the coefficient of average 746 temperature is still significantly negative in the eastern and western regions, in which the influence 747 of average temperature in the east is greater than that in the west, but it doesn't work in the middle. 748 The effect of relative humidity is the most significant in the middle, followed by the east and the 749 weakest in the west. The influence of both average temperature and relative humidity is greater in 750 the coastal areas, which indicating that the role of weather conditions is more important in the 751 coastal areas than inland.

752 Finally, by introducing interaction terms, we explore the moderating effect of diurnal temperature 753 difference, public health measures, and public opinion on the influence of weather conditions on 754 the epidemic to investigate the effects of these factors on the intensity of weather conditions. We 
755 find that the coefficient of the interaction between weather conditions and the high diurnal 756 temperature difference is significantly negative, suggesting that the increase in diurnal temperature 757 differences lead to a stronger impact of weather conditions on the increase in the growth rate of 758 the COVID-19 cases, especially in dry and cold regions, where higher diurnal temperature 759 differences will increase the risk of the spread of the epidemic; the coefficients of the interaction 760 with high public health measures and high social public opinion are both significantly positive, 761 indicating that the improvement of public health measures and social public opinion can weaken 762 the influence of weather conditions on the COVID - 19 confirmed cases growth rate, that is, strict 763 public health measures and sound public opinion can mitigate the adverse effects of cold and dry 764 weather on the spread of the epidemic.

765 Using panel data, this paper applies the two-way fixed effect model of multiple linear regression 766 to explore the causal relationship between weather conditions and the COVID-19 epidemic and 767 takes into account important influencing factors such as human behavior patterns and public health 768 measures, which draws new conclusions. In future research, it can adopt more detailed 769 investigation methods. Under the legal framework of privacy protection, questionnaire surveys can 770 be carried out with patients' consent to draw more accurate conclusions. At the same time, in terms 771 of the mechanism of the role of weather variables, more in-depth interdisciplinary cooperation 772 with epidemiologists is needed to study the specific impact of weather conditions on the 773 survivability of the COVID-19 virus and the immunity of susceptible populations to obtain a 774 clearer picture and compelling conclusions.

\section{$775 \quad$ References}



coronaviruses in the environment and on common touch surfaces and the influence of climatic conditions: A review. Transboundary and emerging diseases, 68(2), 296-312. (2020). Impact of meteorological parameters on COVID-19 pandemic: A comprehensive study from Saudi Arabia. Informatics in medicine unlocked, 20, 100418. syndrome coronavirus. Journal of Infection and Public Health, 13(5), 704-708.

Angrist, J. D., \& Pischke, J. S. (2008). Mostly harmless econometrics: An empiricist's companion. Princeton university press. years of county-level evidence from the United States. American journal of epidemiology, 176(suppl_7), S114788 S122.

Bashir, M. F., Ma, B., Komal, B., Bashir, M. A., Tan, D., \& Bashir, M. (2020). Correlation between climate indicators and COVID-19 pandemic in New York, USA. Science of the Total Environment, 728, 791 138835. temperature on COVID-19 early evolution in Spain. Science of the total environment, 728, 138811.

Brockmann, D., \& Helbing, D. (2013). The hidden geometry of complex, network-driven contagion phenomena. science, 342(6164), 1337-1342.

Brückner, M., \& Ciccone, A. (2011). Rain and the democratic window of opportunity. Econometrica, 79(3), 923-947. 

of Management Science, University of Waterloo. range on human health: a systematic review. International journal of biometeorology, 58(9), 2011-2024. US. Stochastic Environmental Research and Risk Assessment, 34(10), 1675-1680.

Dai, H., \& Zhao, B. (2020, December). Association of the infection probability of COVID-19 with ventilation rates in confined spaces. In Building Simulation (Vol. 13, No. 6, pp. 1321-1327). Tsinghua 811 University Press. 849. 928. in immunology, 11, 1441. 

143315. the COVID-19 pandemic. Nature, 584(7820), 262-267. dispersal of COVID-19. Science of The Total Environment, 736, 139487. 140101. 110355. maximum temperatures and in the diurnal temperature range in Lithuania, Latvia and Estonia in 19512010. Theoretical and applied climatology, 118(1), 57-68. ambient temperature and population size on the transmission rate of the novel coronavirus (COVID-19) in

841 different provinces of Iran. Science of the total environment, 728, 138872.

843 novel coronavirus-infected pneumonia. New England journal of medicine. 

China. Environmental research, 183, 109189. multi-city study in China. Science of the total environment, 726, 138513. UK. relative humidity and temperature. PLoS Pathog, 3(10), e151. Recent Research. International journal of environmental research and public health, 18(2), 396. correlation study between weather and Covid-19 pandemic in Oslo, Norway. Science of the Total Environment, 737, 139659. Higher Temperatures Are Associated With a Marginally Lower Incidence of COVID-19 Cases. Frontiers in public health, 8, 367 . sciences, 129-140. pandemic and WWI. GeoHealth, 4(9), e2020GH000277. 
872 (2021). Weather variability and transmissibility of COVID-19: a time series analysis based on effective reproductive number. Experimental Results, 2.

874

875

876

877

878

879

880

881

882

883

884

885

886

887

888

889

890

891

892

893
Otter, J. A., Donskey, C., Yezli, S., Douthwaite, S., Goldenberg, S., \& Weber, D. J. (2016). Transmission of SARS and MERS coronaviruses and influenza virus in healthcare settings: the possible role of dry surface contamination. Journal of Hospital Infection, 92(3), 235-250.

Owen, S., \& Anil, R. (2009). Mahout in Action, MEAP Edition.

Pahuja, S., Madan, M., Mittal, S., \& Pandey, R. M. (2021). Weather Parameters and COVID-19: A Correlational Analysis. Journal of Occupational and Environmental Medicine, 63(1), 69.

Pan, J., Yao, Y., Liu, Z., Meng, X., Ji, J. S., Qiu, Y., Wang, W., Zhang, L., Wang, W., \& Kan, H. (2021). Warmer weather unlikely to reduce the COVID-19 transmission: An ecological study in 202 locations in 8 countries. Science of The Total Environment, 753, 142272.

Pani, S. K., Lin, N. H., \& RavindraBabu, S. (2020). Association of COVID-19 pandemic with meteorological parameters over Singapore. Science of the Total Environment, 740, 140112.

Paraskevis, D., Kostaki, E. G., Alygizakis, N., Thomaidis, N. S., Cartalis, C., Tsiodras, S., \& Dimopoulos, M. A. (2020). A review of the impact of weather and climate variables to COVID-19: In the absence of public health measures high temperatures cannot probably mitigate outbreaks. Science of the Total Environment, 144578.

Park, J. E., Son, W. S., Ryu, Y., Choi, S. B., Kwon, O., \& Ahn, I. (2020). Effects of temperature, humidity, and diurnal temperature range on influenza incidence in a temperate region. Influenza and other respiratory viruses, 14(1), 11-18.

Polgreen, P. M., \& Polgreen, E. L. (2018). Infectious diseases, weather, and climate. Clinical Infectious Diseases, 66(6), 815-817. 

(2020). COVID-19 transmission in Mainland China is associated with temperature and humidity: a time-series analysis. Science of the total environment, 728, 138778. persistence of SARS-CoV-2 on common surfaces. Virology journal, 17(1), 1-7. seasonal influenza. BMC Public Health, 16(1), 1-14. seasonality. Proceedings of the National Academy of Sciences, 106(9), 3243-3248. dynamics of the COVID-19 outbreak in China. Science of the total environment, 728, 138890. perspectives, 119(4), 439-445.

Tan, J., Mu, L., Huang, J., Yu, S., Chen, B., \& Yin, J. (2005). An initial investigation of the association between the SARS outbreak and weather: with the view of the environmental temperature and its variation. Journal of Epidemiology \& Community Health, 59(3), 186-192. of the American Philosophical Society, 150(1), 86. Biomedica, 17(1), 69-79. 
918 (2013). Climate change and influenza: the likelihood of early and severe influenza seasons following warmer 919 than average winters. PLoS currents, 5. subjects. Physiological reports, 3(7), e12394. Environment, 729, 139051.

\section{Declarations}

- Ethics approval and consent to participate

$936 \quad$ Not applicable

937

- Consent for publication

938

Not applicable

939

- Availability of data and material

940 The datasets during and/or analysed during the current study available from the corresponding author on 941 reasonable request 
- Competing interests

943 The authors declare that they have no competing interests

$944 \bullet \quad$ Funding

945 the National Social Science Foundation of China (Grant No. 13BJY091)

946 the National Natural Science Foundation of China (Grant No.71773083).

$947-$ Authors' contributions

948 Ruofei Lin: Conceptualization, Data curation, Visualization, Formal analysis

949 Xiaoli Wang: Writing- Reviewing and Editing; Validation, Supervision

950 Junpei Huang: Methodology, Software, Writing - Original Draft

$951 \bullet$ Acknowledgements

952 The authors gratefully acknowledge the financial support of the National Social Science Foundation of 953 China (Grant No. 13BJY091) and the National Natural Science Foundation of China (Grant No.71773083).

954 eight severely affected regions in South America. Science of The Total Environment, 744, 140881. 